\title{
Smooth Conjugacy and S-R-B Measures for Uniformly and Non-Uniformly Hyperbolic Systems
}

\author{
R. de la Llave \\ Department of Mathematics, University of Texas, Austin, TX 78712, USA
}

Received June 12, 1991; in revised form May 8, 1992

\begin{abstract}
We give a new proof of the fact that the eigenvalues at corresponding periodic orbits form a complete set of invariants for the smooth conjugacy of low dimensional Anosov systems. We also show that, if a homeomorphism conjugating two smooth low dimensional Anosov systems is absolutely continuous, then it is as smooth as the maps. We furthermore prove generalizations of these facts for non-uniformly hyperbolic systems as well as extensions and counterexamples in higher dimensions.
\end{abstract}

\section{Introduction}

The main purpose of this paper is to present a new proof of the following Theorem 1.1. The new methods we use allow us to make some generalizations, which have not appeared before, among them Theorem 1.2 and Theorem 1.3.

Theorem 1.1. Let $f, g$ be two $C^{k}, k=2,3, \ldots, \infty, \omega$ Anosov diffeomorphisms of a compact two dimensional manifold $M$ (respectively $\sigma_{t}, \psi_{t}$ two Anosov flows of a three dimensional manifold) and $h$ a homeomorphism of $M$ satisfying:

$$
h \circ f=g \circ h
$$

(respectively $h_{\circ} \sigma_{t}=\psi_{t} \circ h$ ). If the Lyapounov exponents at corresponding periodic orbits are the same, then $h \in C^{k-\varepsilon}$.

Theorem 1.2. Let $f, g\left(\right.$ respectively $\left.\sigma_{t}, \psi_{t}\right), h$ be as in Theorem 1.1 and the manifold $M$ be two (respectively three) dimensional. If $h, h^{-1}$ are absolutely continuous with respect to Lebesgue measure then, $h, h^{-1}$ are $C^{k-\varepsilon}$.

Remark. We emphasize that Theorem 1.2 claims only that conjugacies which are both continuous and absolutely continuous are smooth. Since transitive Anosov systems preserving a smooth measure are Bernouilli, one could use Ornstein's theorem to produce absolutely continuous conjugacies between any two Anosov systems with the same metric entropy. They will, nevertheless be discontinuous in general. 
The strategy of all the proofs of Theorem 1.1 in the literature I am aware of consists of two steps: First to prove that the conjugacy is smooth along the leaves of the stable and unstable foliations - with some uniformity. Second, invoke a regularity result that allows to conclude that any function with this property is actually smooth (provided that the foliations have some mild regularity assumptions which will have to be verified for the stable and unstable foliations of Anosov diffeomorphisms).

The regularity lemma needed for the second step was proved first in the case $k \neq \omega$ as Lemma 2.1 of [LMM]; alternate proofs can be found in [HK, Jo1, Jo2, L12]. [Jo1, Jo2] require only properties of the foliations which are well known for Anosov diffeomorphisms, the other proofs require properties which are verified for $k \neq \omega$ in [LMM] Lemma 2.4. The first part of [L12] contains a full treatment of the case $k=\omega$.

The regularity of the conjugating homeomorphism along the leaves was proved by [LM] in the case $k \neq \omega$ and in [L12] for analytic systems. Both papers use, roughly the same geometric idea for this step, the main differences being a different bootstrap of regularity and a different smoothing method in [L12] that could preserve analyticity.

In a recent paper [Po2], M. Pollicott provided an alternative proof of the regularity along leaves based on the theory of zeta functions and Gibbs states that worked for geodesic flows in surfaces of negative curvature. (See also [Po1]).

The first goal of this paper is to simplify further the arguments of [Po2] in such a way that we only use the theory of Gibbs states - so that the method works for transitive Anosov systems, not just geodesic flows. In doing so, we have to remove the use of smooth invariant measures as well as the use of $C^{1+\alpha}$ regularity of stable and unstable foliations, a reuslt which, in spite of several claims in the literature, is not true for Anosov flows in general, as found out by Plante [P1].

The basic idea is to show that, as soon as a conjugacy between two Anosov systems is regular enough so that it has to send $\mathrm{S}-\mathrm{R}-\mathrm{B}$ measures into $\mathrm{S}-\mathrm{R}-\mathrm{B}$ measures, the densities along stable and unstable leaves are also preserved. Since these densities are smooth, the conjugacies should be smooth along the leaves. Performing the argument with enough care will prove that they are uniformly smooth so that the conjugacy is smooth.

We point out that, nevertheless, we inherit some of the features of Pollicott's method, notably that the smooth Livsic's theorem is not invoked and that we do not require a preliminary smoothing. This leads to some technical advantages. For example, we can treat situations in which the hyperbolicity is non-uniform and obtain results that roughly say that except for sets of arbitrarily small measure, the absolutely continuous conjugating homeomorphisms are smooth in the sense of Whitney. In that generality, the regularity result needed is more delicate and we will need to discuss modifications of the proof in [ $\left.\mathrm{J}_{2} 2\right]$ to adapt it to our situation.

We will prove the following theorem which is a generalization of Theorem 1.2 for non-uniformly hyperbolic systems:

Theorem 1.3. Let $f, g$ be two $C^{k}$ diffeomorphisms, $k=2,3, \ldots, \infty$, of a two dimensional manifold $M$ preserving absolutely continuous measures $\mu^{f}, \mu^{g}$ respectively. Assume that, with respect to these measures, $f$ and $g$ are ergodic and have non-zero Lyapounov exponents. If $h$ is a homeomorphism satisfying $h \circ f=g \circ h$, assume either: 
i) Both $h$ and $h^{-1}$ are absolutely continuous with respect to Lebesgue measure, or

ii) The Lyapounov exponents of $\left(f, \mu^{f}\right)$ are equal to the Lyapounov exponents of $\left(g, h * \mu^{f}\right)$.

Then, for every $\varepsilon>0$, we can find a closed set $\Omega_{\varepsilon} \subset M$ whose complement has Lebesgue measure less than $\varepsilon$ such that

$$
\left.h\right|_{\Omega_{\varepsilon}} \in C^{k-\varepsilon},\left.\quad h^{-1}\right|_{\Omega_{\varepsilon}} \in C^{k-\varepsilon} .
$$

We also mention that, as a corollary of the method of proof, we also obtain a version of the smooth Livsic theorem for non-uniform hyperbolic systems. Namely:

Theorem 1.4. Let $f$ be a $C^{k}$ diffeomorphism $k=2,3, \ldots, \infty$ of the compact manifold $M$ which preserves a measure $\mu$ absolutely continuous with respect to Lebesgue. Assume that, with respect to this measure, $f$ does not have a zero Lyapounov exponent. Let $\varphi: M \rightarrow \mathbf{R}$ be a continuous function solving

$$
\varphi \circ f-\varphi=\eta
$$

with $\eta \in C^{k}$. Then, for every $\varepsilon>0$, we can find a closed set $\Omega_{\varepsilon} \subset M$ whose complement has Lebesgue measure less than $\varepsilon$ such that $\left.\varphi\right|_{\Omega_{\varepsilon}} \in C^{k-\varepsilon}$.

Remark. Since the sets in which the function are regular are not open - in typical situations they will be nowhere dense, the statements of regularity of functions are to be understood in the Whitney sense. The main part of the proof will be a regularity lemma for functions defined on such sets. This regularity lemma can be used to generalize the theory of $[\mathrm{LMM}]$ to Axiom A rather than uniformly hyperbolic manifolds. We plan to address these issues in future works.

Remark. Notice that, contrary to the Livsic theorem for uniformly hyperbolic systems, we do not have a result that gives sufficient conditions for the existence of a solution of (1.2) by the vanishing of cocycles on periodic orbits.

Using this, we immediately have:

Corollary 1.5. Let $f, \mu$ be as in Theorem 1.4. If $\mu$ has a continuous density $\rho(x)$, bounded away from zero, then, for every $\varepsilon>0$, we can find a closed set $\Omega_{\varepsilon} \subset M$ whose complement has Lebesgue measure less than $\varepsilon$ such that $\left.\rho\right|_{\Omega_{\varepsilon}} \in C^{k-1-\varepsilon}$.

Proof. By the invariance of $\rho$, we have $\ln (\rho(f(x)))-\ln \rho(x)=\ln (\operatorname{det}((D f(x)))$.

In a subsequent section, we investigate analogues of Theorem 1.1, Theorem 1.2 in higher dimensions.

We prove a rigidity statement showing that conjugacies smoother than a critical regularity are as smooth as the maps. (For the partially hyperbolic case, the regularity statement requires to exclude a set of arbitrarily small measure.)

We also show that in dimension greater than or equal to four, in general, there are no local invariants around periodic orbits whose vanishing implies smooth conjugacy. Indeed, we construct examples of systems which are topologically conjugated and such that, around every periodic orbit, we can find neibghbourhoods which are smoothly conjugated. Nevertheless, the systems are not $C^{k}$ conjugated for some $k$. These results show that the hypothesis of a minimal regularity in the rigidity results cannot be dropped. 


\section{Acknowledgements}

I thank M. Pollicott for showing me his paper [Po2] prior to publication as well as for very enlightening discussions. A version of the first chapters of this paper was typed at I.H.E.S. September 1988. It is a pleasure to thank D. Ruelle for his advice. I would also like to thank J-L. Journé for explaining [Jo2] to me and for several discussions. My work has been supported by N.S.F. grants.

\section{Preliminary Results and Notation}

In this section we set the notation and recall without proof - but with precise references to the literature - several facts which we will need later. In this preliminary section we will discuss only Anosov systems. Some, but not all, of the results we discuss have an analogue for non-uniformly hyperbolic systems. Some of these generalizations will become useful in Sect. 5, where we will discuss them in more detail.

We recall that an Anosov diffeomorphism $f$ in a manifold $M$ leaves invariant two foliations $W^{s(f)}, W^{u(f)}$, whose leaves are characterized by

$$
\begin{aligned}
& W_{x}^{s(f)}=\left\{y \mid d\left(f^{n}(y), f^{n}(x)\right) \rightarrow 0, n \rightarrow+\infty\right\}, \\
& W_{x}^{u(f)}=\left\{y \mid d\left(f^{-n}(y), f^{-n}(x)\right) \rightarrow 0, n \rightarrow+\infty\right\} .
\end{aligned}
$$

For a flow $\sigma$, besides the stable and unstable foliations defined as above, we can also consider the center-stable and center-unstable invariant foliations $W^{\operatorname{sc}(\sigma)}, W^{\mathrm{uc}(\sigma)}$ which include the direction of the flow in the leaves. (When there is no possibility of confusion, we will drop the index that tells which diffeomorphism or flow the foliations correspond to.) The local pieces of the foliation will be denoted by:

$$
\begin{aligned}
W_{x}^{\mathrm{s}(f), \delta} & =\left\{y \mid d\left(f^{n}(y), f^{n}(x)\right)<\delta, n>0\right\} \\
W_{x}^{\mathrm{u}(f), \delta} & =\left\{y \mid d\left(f^{-n}(y), f^{-n}(x)\right)<\delta, n>0\right\} .
\end{aligned}
$$

The definition makes it clear that a topological conjugacy between two different Anosov diffeomorphisms (or flows), sends the leaves of each of the foliations for one system into the leaves of the corresponding foliation of the other.

These foliations, nevertheless, have several regularity properties which are more than topological. We will use that if the diffeomorphism or flow is $C^{k}$, $k=1 \ldots \infty, \omega$, the leaves are $C^{k}$ and that the jets of the leaves are Hölder.

The definition of Hölder continuity of jets can only be done using patches of coordinates. We will denote by $J_{x}^{k}(\Phi, N)$, the $k$-jet of the function $\Phi$ with range in the manifold $N$ at a point $x$. (We will use only $J_{x}^{k}$ if the function and the space are understood.) When $k$ is a finite number $\left\|J_{x}^{k}\right\|$ can be defined naturally if there is a metric on the manifolds. When $k=\omega$, we can take several norms, depending on an parameter $\eta>0$, roughly, the size of the analyticity domain, $\left\|J_{x}^{\infty}\right\|_{\eta}=\sum_{i=0}^{\infty}\left\|J_{x}^{k}\right\| \eta^{k} / k !$

In a coordinate patch, we can identify a jet with a sequence of $i$-linear symmetric forms, $i=1, \ldots, k$ in $\mathbf{R}^{n}$ - the expressions of the derivatives in this 
coordinate system - and subtract them. The case $k=\infty$ we can define not a norm but a distance $d\left(J_{x}^{\infty}, J_{y}^{\infty}\right)=\sum_{i=0}^{\infty} \frac{\left\|J_{x}^{k}-J_{y}^{k}\right\|}{1+\left\|J_{x}^{k}-J_{y}^{k}\right\|} 2^{-k}$.

This allows us to define continuity and Hölder continuity of jets and give the space of Hölder jets a norm

$$
\left\|J^{k}\right\|_{C^{\alpha}}=\sup \left\|J_{x}^{k}-J_{y}^{k}\right\| / d(x, y)^{\alpha} .
$$

This definition depends on the choice of coordinate patches. It is well known that, when $k \neq \omega$, even if the norm changes when we change the coordinate patches, all the norms one constructs in this way are equivalent. When $k=\omega$ it is not difficult to show that if we choose another analytic coordinate patch, given $\eta>0$ we can find $\eta^{\prime}>0$ such that $\left\|J_{x}^{\infty}\right\|_{\eta} \leqq C\left\|J_{x}^{\infty}\right\|_{\eta^{\prime}}^{\prime}$.

For many purposes, the use of one equivalent norm in place of another causes no difficulty. One exception is contraction arguments. In many of the cases, the fact that the operators we need are contractions will require that the patches we take have sufficiently small diameter. Such assumptions will appear in a finite number of places and can be met.

We will also need some regularity properties of stable and unstable foliations. We refer to [HPS, HP, S, Fe1, Fe2] for the case of Anosov systems and to [Pe, Ru2, FHY, PS2] for the case of non-uniformly hyperbolic systems which we will discuss in Sect. 5.

The stable manifold theorem for Anosov systems that we will use reads:

Theorem 3.1. Let $f$ be a $C^{k}$ Anosov diffeomorphism (respectively $\sigma_{t}$ an Anosov flow) $k=1,2, \ldots, \infty, \omega$, then, $W_{x}^{\mathrm{s}, \delta}, W_{x}^{\mathrm{u}, \delta}\left(\right.$ respectively $\left.W_{x}^{\mathrm{cs}, \delta}, W_{x}^{\mathrm{cu}, \delta}\right)$ are $C^{k}$ manifolds. The $k$-jets of the leaves are Holder with respect to $x$.

Following [LMM] we define regularity classes related to Anosov systems. (The analytic case was discussed in more detail in [L12]; our present definition is not the same as that in this paper, but for all purposes we consider it is equivalent.)

We say that a function $\phi: M \mapsto \mathbf{R}$ is in $C_{\mathrm{s}(f)}^{k}, k=1,2, \ldots, \infty$ when the restriction of $\phi$ to the leaves of $W^{\mathrm{s}(f)}$ is $C^{k}$ and the $k$-jets are $C^{0}$ on the manifold. Similarly, we say that it is in $C_{\mathrm{s}(f)}^{k, \alpha}$ if the restriction of $\phi$ to the leaves of $W^{\mathrm{s}, f}$ is $C^{k, \alpha}$, that is it is $k$ times differentiable and the $k^{\text {th }}$ derivative is $C^{\alpha}$. Furthermore, we require that the $k$-jets are continuous on the manifold. We say that $\phi$ is in $C_{\mathrm{s}(f)}^{\omega}$ when there is an analytic coordinate patch and a number $\eta>0$ such that the mapping $x \mapsto J_{x}^{\infty}\left(\left.\phi\right|_{W_{x}^{\mathrm{s}}}, \mathbf{R}\right)$ is continuous if we topologize the space of jets with the norm \|\|$_{\eta}$ defined above using the set of coordinates. All these spaces have associated natural norms that make them Banach spaces. We will denote them by \|\|$_{C_{\mathbf{s}(f)}^{k}}$ and if $f$ is understood we will omit it.

Using standard regularity theory of the stable and unstable foliations it is easy to show that this definition is consistent. Moreover, if $r \leqq k, C^{r} \subset C_{\mathrm{s}}^{r} \cap C_{\mathrm{u}}^{r}$.

Since these regularity classes are well adapted to the dynamics, they play an important role in many discussions of regularity. The regularity in these classes can be related to more conventional regularity classes. For example, the same argument that proves that a function having continuous partial derivatives is $C^{1}$ establishes that $C_{\mathrm{s}}^{1} \cap C_{\mathrm{u}}^{1}=C^{1}$. A deeper result is the following.

Lemma 3.2. In the conditions above: $k=2, \ldots, \infty, \omega, r \leqq k$,

$$
C_{\mathrm{s}}^{r} \cap C_{\mathrm{u}}^{r} \subset C^{r-\varepsilon} .
$$


This result is really a basic regularity result about functions restricted to foliations and can be generalized to foliations satisfying some regularity properties (that will have to be verified for the invariant foliations of Anosov systems).

Remark. We observe that, even in the case where the foliations are trivial, $C_{\mathrm{s}}^{r} \cap C_{\mathrm{u}}^{r} \neq C^{r}$. An example, due to Yudovitch can be found in [Kr]. From the point of view of these regularity results, the most natural spaces are the Lipschitz spaces $\mathrm{Lip}_{\alpha}$ or the $\Lambda_{\alpha}$ spaces. Unfortunately, they are not easy to use in dynamical systems since they do not behave well under composition. So, parts of our arguments have to rely on $C^{k}$ for $k \in \mathbf{N}$ even if for the regularity arguments, the most natural spaces would be in other spaces. The techniques we use to prove these results when the foliations are non-trivial rely on converse Taylor methods and hence $\operatorname{Lip}_{\alpha}$ seem more natural.

The proofs of Lemma 3.2 in [LMM, HK, L12] use the following properties of Anosov foliations:

Lemma 3.3. Let $f$ be a $C^{k}$ Anosov diffeomorphism $k=1, \ldots, \infty, \omega$ in the $n$ dimensional manifold $M$. One can choose mappings $\Lambda_{u}$ from sufficiently small neighborhoods in $M$ to $\mathbf{R}^{n}$ in such a way that:

1) $\Lambda_{\mathrm{u}}\left(W_{x}^{\mathrm{u}}\right)=\left\{\left(x_{1}, \ldots, x_{n}\right) \mid x_{1}=c_{1}, \ldots, x_{\mathrm{u}}=c_{\mathrm{u}}\right\}$.

2) The mapping $\Lambda^{\mathrm{u}}$ is absolutely continuous and its Jacobian agrees locally with a function in $C_{\mathbf{u}}^{k-1}$.

This lemma is proved for $k \neq \omega$ in [LMM]. For $k=\omega$ it is proved in [L12].

There are other proofs based on another idea [Jo1, Jo2] that only use continuity of the foliations - or Hölder continuity. Unfortunately, these proofs do not work to recover analyticity. We will discuss in more detail and strengthen the proof of [ Jo2] in Sect. 5 since this proof seems to be quite useful in the context of partially hyperbolic systems.

We will also recall some facts about the $\mathrm{S}-\mathrm{R}-\mathrm{B}$ (Sinai-Ruelle-Bowen measures) $\mu_{+}, \mu_{-}$. (As usual we will use $\mu^{(f)}$ if there is risk of confusion.) These measures can be characterized uniquely by different properties that we now describe. It is a very deep theorem that, for transitive $C^{1+\alpha}$ Anosov systems, each of these properties implies the others. We refer to [Si2, $\mathrm{Ru}, \mathrm{BR}, \mathrm{B}]$ for the proofs of these equivalences. Some of these definitions and equivalences extend to non-uniformly hyperbolic systems. We will use some of these extensions in Sect. 5, where we will discuss them in more detail.

Property 3.4. For any continuous function $\phi: M \mapsto \mathbf{R}$, the following identities hold for almost all $x$ with respect to Lebesgue measure:

$$
\lim _{N \rightarrow \infty} \frac{1}{N} \sum_{n=0}^{N-1} \phi\left(f^{n}(x)\right)=\int \phi d \mu_{+} ; \quad \lim _{N \rightarrow \infty} \frac{1}{N} \sum_{n=0}^{N-1} \phi\left(f^{-n}(x)\right)=\int \phi d \mu_{-} .
$$

For flows, there are similar definitions substituting for the sum in $n$, an integral in the continuous time. We observe that, by the Birkhoff ergodic theorem, if there is an absolutely continuous invariant measure it has to agree with the $\mathrm{S}-\mathrm{R}-\mathrm{B}$ measures and be ergodic.

The following definitions were introduced in [Si2], where they were shown to be quite useful. We have followed the notation of [LY1], since in Sect. 5 we will use some results of this paper. 
Definition 3.5. Given a manifold $M$, a Borel measure $\mu$ and $a$ foliation $W$ on it and we say that the measurable partition $\xi$ is subordinate to the foliation $W$ whenever for $\mu$ a.e. we have:

1) $\xi(x) \subset W_{x}$.

2) $\xi(x)$ contains an open subset of a neighborhood of $x$ in $W_{x}$ in the submanifold topology.

Definition 3.6. We say that $\xi$ is increasing and subordinate to $W^{\mathrm{u}(f)}$ if it is subordinate to $W^{\mathrm{u}(f)}$ and

1) $f \xi$ refines $\xi$.

2) $\bigvee_{n=0}^{\infty} f^{-n} \xi$ is the partition into points.

3) The biggest $\sigma$-algebra contained in $\bigcap_{n=0}^{\infty} f^{n} \xi$ is the $\sigma$-algebra whose elements are unions of $W^{\mathrm{u}}$ manifolds.

Analogous definitions can be made for $W^{\text {s(f) }}$. It is also possible to generalize the definition of increasing for flows by taking the same definitions as above with $f$ replaced by a time one map.

Remark. We observe that if $f, g$ (respectively $\left.\sigma_{t}, \psi_{t}\right), h$ are as in (1.1) and $\xi$ is a partition subordinate (increasing) to $W^{\mathrm{u}(f)}$, then, $h(\xi)$ - the partition whose elements are the images under $h$ of the elements of $\xi$-is subordinate (increasing) to $W^{\mathrm{u}(g)}$.

Definition 3.7. We say that $\mu_{x}^{\xi}$ is the measure conditioned by the partition $\xi$ if for every measurable $A \subset M, x \mapsto \mu_{x}^{\xi}(A)$ is measurable with respect to the $\sigma$-algebra generated by $\xi$ and $\mu(A)=\int \mu_{x}^{\xi}(A) \mu(d x)$.

Clearly, if $\xi_{1}$ refines $\xi_{2}$ the conditional measures $\mu_{x}^{\xi_{2}}$ are absolutely continuous with respect to $\mu_{x}^{\xi_{1}}$.

A very important characterization of S-R-B measures $\mu_{+}$and $\mu_{-}$is the following:

Property 3.8. For every measurable partition $\xi$ subordinate to $W^{\mathrm{u}}\left(\right.$ respectively $\left.W^{\mathrm{s}}\right)$, $\mu_{+x}^{\xi}$ (respectively $\mu_{-x}^{\xi}$ ) is absolutely continuous with respect to the Lebesgue measure induced by the Riemann metric on $W_{x}^{\mathrm{u}}$ (respectively $W_{x}^{\mathrm{s}}$ ).

We will denote by $\omega_{+x}^{\mathrm{u} \xi}$ the density of $\mu_{+x}^{\xi}$ with respect to the induced Lebesgue measure $W_{x}^{\mathrm{u}}$ and analogously for $\mu_{-}$.

When we have partitions which are increasing and subordinate to $W^{\mathrm{u}}$, there is a canonical way of obtaining "maximal" conditional measures.

Notation. For a diffeomorphism $f$, we will denote by $\Psi_{n}^{\mathrm{u}(f)}(x)$ the Jacobian of $f^{n}$ restricted to the unstable foliation and evaluated at $x$. Similarly for unstable and for flows. For flows, $n$ above will be a real variable rather than integer and $\Psi_{n}^{\mathrm{u}(\sigma)}(x)$ will denote the unstable Jacobian of the time- $n$ map. In general, the Jacobian may depend on the metric. Nevertheless, for a periodic orbit $x$ of period $n, \Psi_{n}^{\mathrm{u}(f)}(x)$ is independent of the metric.

Notice that the chain rule and the invariance of the foliation give:

$$
\Psi_{n}^{\mathrm{u}(f)}(x)=\prod_{i=0}^{n-1} \Psi_{1}^{\mathrm{u}(f)}\left(f^{i}(x)\right) .
$$



orbit.

In particular, the stable, unstable Jacobians are the same for all points in the

Theorem 3.9. Let $f$ be a Anosov diffeomorphism (respectively $\sigma_{t}$ an Anosov flow) $\xi$ an increasing partition subordinate to $W^{\mathrm{u}}$. Denote $\xi_{N}=\bigvee_{n=0}^{N} f^{-n} \xi$ (respectively $\left.\xi_{N}=\bigvee_{n=0}^{N} \sigma_{-n} \xi\right)$ then for $\mu_{+}$almost all $x$, for all $y$ in $W_{x}^{\mathrm{u}}, \lim _{N \rightarrow \infty} \omega_{+x}^{\mathbf{u} \xi_{N}}=\omega_{+x}$ exists a.e. with respect to Lebesgue measure. Moreover, for $\mu_{+}$almost all $x$ we have for all $y, z$ in $W_{x}^{\mathrm{u}}$ :

$$
\ln \omega_{+z}(x)-\ln \omega_{+z}(y)=\lim _{n \rightarrow \infty}\left(\ln \left(\Psi_{-n}^{\mathrm{u}}(x)\right)-\ln \left(\Psi_{-n}^{\mathrm{u}}(y)\right) \equiv \ln \Delta(x, y) .\right.
$$

Analogous results hold for $\mu_{-}$and the stable foliation.

Remark. Notice that in (3.3) the right-hand side does not depend on the partition nor on the point $x$. Hence, the conditional densities $\omega_{+}$are unique up to multiplicative factors and their quotients are natural geometric objects.

Proof. This result was - in other slightly different language - proved in [Si2] along the lines of the proof that the measures $\mu_{+}$and $\mu_{-}$exist (Theorem 4, p. 28. ff. for diffeomorphisms, p. $44 \mathrm{ff}$. for flows). In the language of [Si2], $\Psi_{1}^{\mathrm{u}(f)}$ is the potential for the Gibbs measure $\mu_{+}$.

If one assumes that the measures exist and are absolutely continuous, one can establish (3.3) by looking at the formula for the change of variables and using the theorem of differentiation of integrals. This is done in [LY1] (Proof of Lemma 6.1.1, pp. 553, ff.) for diffeomorphisms with positive Lyapounov exponents with respect to the measure $\mu$.

It is an important fact that for Anosov diffeomorphisms and flows and nonuniformly hyperbolic systems, one can find increasing partitions subordinate to the stable and unstable manifolds. It is even possible to find partitions that satisfy extra properties. For Anosov systems - both flows and diffeomorphisms - it is possible to find Markov partitions [B] that, besides being subordinate, enjoy other properties. For non-uniformly hyperbolic systems, the construction of increasing partitions subordinate to the stable and unstable manifolds can be found in [LS, LY1] for diffeomorphisms.

There is still another characterization of S-R-B measures that emphasizes the role played by the Lyapounov exponents at periodic orbits. Given a periodic orbit $o$ we will denote by $T(o)$ the minimal period and by $\delta_{o}$ the natural measure of mass $T(o)$ invariant under time evolution on the orbit. For diffeomorphisms, $\delta_{o}$ will be the counting measure on the points belonging to the orbit. For flows, it will be the length of time spent in an interval.

We will denote by $\operatorname{Per}_{-}^{(\sigma)}(n)$ the set of periodic orbits with period in $[n, n+1)$ of the flow $\sigma$. For diffeomorphisms it is just the points of minimal period $n$.

\section{Property 3.10.}

$$
\lim _{n \rightarrow \infty} \frac{1}{Z_{n}} \sum_{o \in \operatorname{Per}-(n)}\left(\Psi_{o}^{\mathrm{u}(f)}\right)^{-1} \delta_{o}=\mu_{+},
$$

where $Z_{n}$ is a normalization factor to make the total mass of the right-hand side one and the limit is understood in the weak* sense. 
Proof. The fact that the measure can be approximated by the measure at periodic orbits can be proved very easily using the thermodynamic formalism after using Markov partitions and observing that $\mu_{+}$is the equilibrium state for the unstable Jacobian and $\mu_{-}$for the stable. (See e.g. [Ru] for Axiom A diffeomorphisms, [BR] for Axiom A flows.)

Once one uses Markov partitions and thermodynamic formalism, there is little difference between diffeomorphisms and flows and the dimension of the space plays no role. The use of Markov partitions for Anosov flows to apply to the thermodynamic formalism is worked out in detail in [Si2], pp. 42-48. Some results are claimed only for dimension 3 because at the time that [Si2] was written, Markov partitions were known to exist for flows only when manifolds on which they were defined were of dimension 3. Full details about how to construct Markov partitions for the Anosov diffeomorphism and flows in any number of dimensions were worked out in [Bo, Ra]. One geometric, i.e. without Markov partitions, pedagogical proof of Property 3.10 for Anosov diffeomorphisms can be found in [Ne].

\section{Proof of Theorems 1.1 and 1.2}

In this section, we use the previously quoted results and prove Theorem 1.1 and Theorem 1.2.

Lemma 4.1. Let $f, g$ be $C^{2}$ transitive Anosov diffeomorphisms (respectively $\sigma_{t}, \psi_{t}$ transitive Anosov flows) of an $n$-dimensional manifold $M, h$ a homeomorphism of $M$ satisfying: $h \circ f=g \circ h$ (respectively $h \circ \sigma_{t}=\psi_{t} \circ h$ ). If the unstable Jacobian at corresponding periodic orbits is the same then: $h^{*} \mu_{+}(f)=\mu_{+}(g)$ (respectively $\left.h^{*} \mu_{+}(\sigma)=\mu_{+}(\psi)\right)$.

Proof. Observe that - both for diffeomorphisms and flows - a conjugating homeomorphism carries the natural measures on orbits to natural measures on orbits. Using this observation and the hypothesis about Jacobians at periodic orbits (to simplify the notation we only perform the calculation for diffeomorphisms):

$$
h^{*} \sum_{o \in \operatorname{Per}_{-(f)}^{(n)}}\left(\Psi_{o}^{\mathrm{u}(f)}\right)^{-1} \delta_{x}=\sum_{o \in \operatorname{Per}_{-}^{(f)}(n)}\left(\Psi_{o}^{\mathrm{u}(f)}\right)^{-1} \delta_{h(x)}=\sum_{p \in \operatorname{Per}^{(g)}(n)}\left(\Psi_{p}^{\mathrm{u}(g)}\right)^{-1} \delta_{p} .
$$

Observe also that the hypothesis about unstable Jacobians also implies that the partition functions corresponding to the two sums are the same. Dividing by them and taking limits using Property 3.10, proves the lemma.

Lemma 4.2. Under the hypothesis of the previous theorem, the restriction of $h$ to a leaf of the unstable foliation is absolutely continuous - with respect to Lebesgue measures in the domain and the range. Moreover, we have:

$$
\int_{A} \omega_{+x}^{(f)}(y) d y=\int_{h(A)} \omega_{+h(x)}^{(g)}(y) d y,
$$

where $d x$ is the Lebesgue measure induced by the metric on $W_{x}^{\mathrm{u}}$ and $A$ is any subset in $W_{x}^{\mathrm{u}}$.

Proof. Equation (4.1) is just the translation in coordinates of the fact that if the Gibbs measures are transformed into one another, so are the restrictions when the 
partitions are also transformed with $h$. The absolute continuity is an easy consequence of this formula because if $h(A)$ had measure zero, the right-hand side should be zero, which can only happen if the measure of $A$ is zero because the density is positive.

The following lemma is perhaps the most crucial step in the argument. We want to prove regularity properties of the restricted densities.

Lemma 4.3. Under the assumptions and notations of Theorem 1.1 and Theorem 1.2, let $\Delta_{\mathrm{u}}(x, y)$ be as in (3.3). For each point $x \in M$ introduce the function $\Delta_{\mathbf{u}}^{x}(y)=\Delta_{\mathbf{u}}(x, y)$. Then, for $r=1, \ldots, k-1,\left\|J_{y}^{r}\left(\left.\Delta_{\mathbf{u}}^{x}\right|_{W_{x}^{u}}, \mathbf{R}\right)\right\|$ is bounded uniformly in $x, y \in W_{x}^{\mathrm{u}, \delta}$. Moreover, if $r \leqq k$ is finite, there will be a uniform modulus of continuity for the functions defined on $W_{x}^{\mathrm{u}, \delta} y \mapsto J_{y}^{r}\left(\Delta_{x}^{\mathrm{u}}(y)\right)$. When $k=\omega$, we can obtain uniform bounds in \|\|$_{\eta}$.

Corollary 4.4. For a $C^{k}$ Anosov diffeomorphism, the densities of the restriction to stable or unstable foliations of the $\mathrm{S}-\mathrm{R}-\mathrm{B}$ measures are $C^{k-1}$.

Remark. Notice that we are not claiming that the function $\Delta(x, \cdot)$ is in $C_{\mathrm{u}}^{k}$. As a matter of fact it will come out of the proof that it cannot be extended in a continuous way to the whole manifold.

Proof. For the sake of simplicity, we will only consider the case of diffeomorphisms. Using (3.2), we can write

$$
\ln \left(\Psi_{-n}^{\mathrm{u}}(x)\right)-\ln \left(\Psi_{-n}^{\mathrm{u}}(y)\right)=\sum_{i=0}^{n-1}\left[\ln \Psi_{-1}^{\mathrm{u}}\left(f^{-i}(x)-\ln \Psi_{-1}^{\mathrm{u}}\left(f^{-i}(y)\right)\right] .\right.
$$

So that to prove that the limit converges uniformly it is sufficient to show, by Weierstrass' $M$ test, that the terms of the sum can be bounded uniformly by a convergent sequence.

Using the fact that $f^{-1}$ is contracting when restricted to a $W_{x}^{\mathrm{u}}$ uniform factor less than 1 and that the $\Psi_{-1}^{\mathrm{u}}(x)$ is uniformly Lipschitz (being Hölder would suffice) it is easy to show that the sum is bounded by an exponentially decreasing sequence.

We claim that if we take derivatives with respect to $y$ term by term in the sum, we will obtain a series that can be bounded uniformly by a decreasing exponential. By Weierstrass' $M$ test, the series of derivatives converges uniformly and is the derivative of the sum. The estimates needed to obtain the exponential bounds are the same as in [LMM], Lemma 2.2, p. 573, ff. Namely, it suffices to show that, using the chain rule, we can write $\left(\frac{d}{d y}\right)^{n} \ln \Psi_{-1}^{\mathrm{u}} \circ f^{-i}(y)$ as a sum containing not more than $n$ ! terms, each of which contains not more than $i^{n}$ factors. These factors consist of derivatives of order at most $n$ of $\ln \Psi_{-1}^{\mathrm{u}} \circ f^{-i}(y)$ and of $f^{-1}$. Moreover, each term contains at least $i-n$ factors of the form $f^{-1}\left(f^{-j}(x)\right)$. Since the latter can be bounded by a constant strictly smaller than 1 , and the derivatives of $\ln \Psi_{-1}^{\mathrm{u}}$ can be bounded uniformly. The result follows.

We point out that we will prove in more detail a more general result in Sect. 5. The statement we will prove will include information about the modulus of continuity.

The proof for $k=\omega$ is somewhat easier. We just observe that the functions we are considering admit complex extensions. The stable manifolds are analytic 
immersions of the disk into the manifold and, hence, can be extended to a complex extension of the disk. Likewise the Jacobians of $f$ and its restriction can also be considered in a complex extension. The size of the complex extensions can be bounded uniformly over the manifold and the supremum in the complex extension can be bounded uniformly as well as the Lipschitz constant for a sufficiently small extension. The same argument used to show convergence can be used to show uniform convergence of the sum in a complex extension and, hence, analyticity of the sum.

Remark. The proof that the function $\Delta$ is Lipschitz when $f$ is $C^{2}$ can be found in [LY1], where it is also stated without proof that it is $C^{1}$. The function $\Delta$ is very similar to the function $\Gamma$ in [L12]. The arguments used there to study the regularity work in our situation.

Lemma 4.5. Let $f, g$ be Anosov diffeomorphisms of a two dimensional manifold $(\sigma, \psi$ Anosov flows on a three dimensional manifold) $h, k$ be as in Theorem 1.1 and Theorem 1.2. Assume moreover that $W^{u}$ has one-dimensional leaves. If $h$ verifies $h^{*} \mu_{+}(f)=\mu_{+}(g)$, then $h \in C_{\mathbf{u}}^{k(f)}$.

Remark. Analogous results hold for the stable manifold and for flows the proofs are the same.

Proof. When the unstable foliation has one dimensional leaves, we can parametrize patches of the unstable leaves by the Riemann length $s$. Then, (4.1) can be rewritten (identifying in an obvious way functions of the manifold and functions of the coordinates):

$$
\int_{0}^{s} \omega_{0}^{\mathrm{u} \xi(f)}\left(s^{\prime}\right) d s^{\prime}=\int_{h(0)}^{h(s)} \omega_{h(0)}^{\mathrm{u} h(\xi)(g)}\left(s^{\prime}\right) d s^{\prime} .
$$

This is a differential equation in integral form. Using the monotonicity of $h$, we can see it is equivalent to

$$
\omega_{0}^{\mathrm{u} \xi(f)}(s)=h^{\prime}(s) \omega_{h(0)}^{\mathrm{u} h(\xi)(g)}(h(s)) .
$$

By Lemma 4.3, the coefficients are in $C^{k}$. By regularity theory of ordinary differential equations $h$ will be $C^{k}$ when restricted to a leaf of the unstable foliation. Moreover, since the function defining the differential equation is uniformly $C^{k}$, $h$ will be uniformly $C^{k}$ when restricted to any leaf. Now we need to conclude that $h \in C_{\mathrm{u}}^{k}$. This can be done by a closed graph argument. Take a sequence of patches of leaves converging to another patch. The restriction of $h$ to those patches are uniformly bounded in $C^{k}$. Hence we can get a subsequence converging in the $C^{k-\varepsilon}$ sense. Since we know that $h$ extends continuously, the limit of this subsequence should be the restriction of $h$. Hence, there is a $C^{k-\varepsilon}$ limit of the full sequence.

Out of these results it is very easy to complete the proof of Theorem 1.1.

By the hypothesis on the eigenvalues, we can apply Lemma 4.1 and, since the manifold is low dimensional, the stable and unstable manifold will have onedimensional leaves so we can apply Lemma 4.5. Hence $h$ should be in $C_{\mathrm{s}}^{k} \cap C_{\mathrm{u}}^{k}$ and, we just apply the regularity results mentioned before.

This finishes the proof of Theorem 1.1. 
Remark. Equation (4.2) was derived by brute force in [L12]. The motivation was to get a method to bootstrap regularity more robust than that of [L11]. The source of inspiration was a very similar calculation done in [Pr]. The argument presented in [L12] goes through for all one-dimensional foliations even if there is no natural invariant measure. This plays a role in [L13].

Remark. The use of smooth invariant measures to prove smoothness of conjugacies in one dimension appears also in [He, SS].

Remark. Results very similar to Lemma 4.2 could have been derived by the scattering method of [L11]. One essential ingredient of this method is a previous smoothing, which forces some extra hypothesis. The method in [LM] requires that the leaves of the stable and unstable manifolds are one-dimensional, the method used in [L12] would require that the Anosov systems we consider are in the same connected component of the set of Anosov diffeomorphisms.

Now, we proceed to complete the proof of Theorem 1.2.

Lemma 4.6. Let $f, g$ (respectively $\sigma_{t}, \psi_{t}$ ), $h$ be as in Lemma 4.1. If $h, h^{-1}$ are absolutely continuous with respect to Lebesgue measure then, $h^{*} \mu_{+}(f)=\mu_{+}(g)$ (respectively $h^{*} \mu_{+}(\sigma)=\mu_{+}(\psi)$ ).

Proof. For any continuous function $\phi$ we have by the conjugacy equation:

$$
\frac{1}{N} \sum_{n=0}^{N-1} \phi\left(g^{n}(h(x))\right)=\frac{1}{N} \sum_{n=0}^{N-1} \phi \circ h\left(g^{n}(x)\right) .
$$

By the absolute continuity of $h$ and Property 3.4, there is one $x$ for which the two limits exist and take the limit in (3.1). Then we have $\int \phi \circ h d \mu_{+}(f)=\int \phi d \mu_{+}(g)$, analogously for $\mu_{-}$.

Using this lemma, Lemma 4.5 and the regularity results we have proved Theorem 1.2.

\section{Proof of Theorem 1.3}

The proof of Theorem 1.3 is more technical than the proof of the other theorems. What we will do is to show that the partial regularity results along leaves hold for large sets that have certain geometric structure, and then we will prove an analogue of Lemma 3.2 for those sets.

We will start by recalling some facts from the theory of non-uniform hyperbolic systems. Complete proofs can be found in [Pe, Ru2, Ka, FHY, LY1, LY2].

Using Osledec's ergodic theorem and some auxiliary constructions explained in detail in $[\mathrm{Pe} 2, \mathrm{Ru}, \mathrm{Ka}]$ it is possible to prove the following theorem (we have followed the statement of [Ka] except for some minor modifications):

Theorem 5.1. Let $f$ be a diffeomorphism as in Theorem 1.3. Let $\mu$ be any ergodic invariant measure and let $\gamma_{+}, \gamma_{-}$be its Lyapounov exponents $\gamma_{+}>0, \gamma_{-}<0$. Given any $\varepsilon>0$, we can find a set $\Omega_{\varepsilon}$ and a number l such that $\mu\left(M-\Omega_{\varepsilon}\right) \leqq \varepsilon, \Omega_{\varepsilon}$ is closed and, for each $x \in \Omega_{\varepsilon}$ we have: There exists a decomposition $T_{x} M=E_{x}^{\mathrm{s}} \oplus E_{x}^{\mathrm{u}}$ in such a way that, for all $n \in \mathbf{Z}^{+}, m \in \mathbf{Z}$, 
1) If $v \in D f^{m}(x) E_{x}^{\mathrm{s}}$,

$$
\begin{aligned}
\left\|D f^{n}\left(f^{m}(x)\right) v\right\| & \leqq l \exp \left(n \gamma_{-}\right) \exp ((n+|m|) \varepsilon)\|v\|, \\
\left\|D f^{-n}\left(f^{m}(x)\right) v\right\| & \geqq l^{-1} \exp \left(-n \gamma_{-}\right) \exp (-(n+|m|) \varepsilon)\|v\| .
\end{aligned}
$$

2) If $v \in D f^{m}(x) E_{x}^{\mathrm{u}}$,

$$
\begin{aligned}
\left\|D f^{n}\left(f^{m}(x)\right) v\right\| & \geqq l \exp \left(n \gamma_{+}\right) \exp (-(n+|m|) \varepsilon)\|v\|, \\
\left\|D f^{-n}\left(f^{m}(x)\right) v\right\| & \leqq l^{-1} \exp \left(-n \gamma_{+}\right) \exp (+(n+|m|) \varepsilon)\|v\| .
\end{aligned}
$$

3) If $\gamma(x)$ denotes the angle between $E_{x}^{\mathrm{s}}, E_{x}^{\mathrm{u}}$, we have

$$
\gamma\left(f^{m}(x)\right) \geqq l^{-1} \exp (-\varepsilon|m|) .
$$

4) The decomposition $T_{x} M=E_{x}^{\mathrm{s}} \oplus E_{x}^{\mathrm{u}}$ is continuous restricted to $\Omega_{\varepsilon}$.

For each of the points in $\Omega_{\varepsilon}$ it is possible to prove an invariant manifold theorem (see any of the references above, in particular [FHY]). The important point is that, since the hyperbolicity constants are uniform in $\Omega_{\varepsilon}$ we can conclude that the regularity properties of the invariant manifolds are uniform for all points in $\Omega_{\varepsilon}$.

Theorem 5.2. Let $x$ be in $\Omega_{\varepsilon}$ as before, $m$ be an integer and $d_{\mathrm{s}}$ denote the dimension of $E_{x}^{\mathrm{s}}$. If $f$ is $C^{k}, k=2, \ldots, \infty, \omega$. We can find $C^{k}$ mappings $\mathscr{W}_{f^{m}(x)}^{\mathrm{s}}:[-1,1]^{d_{\mathrm{s}}} \mapsto M$ in such a way that:

1) For all $r \leqq k, r \in \mathbf{Z}\left\|\mathscr{W}_{f^{m}(x)}^{\mathrm{s}}\right\|_{C^{r}} \leqq l_{m} \exp \left(\varepsilon C_{r} m\right)$.

2) $\mathscr{W}_{f^{m}(x)}^{\mathrm{s}}(0)=f^{m}(x)$.

If we denote by $W_{f^{m}(x)}^{\mathrm{s}, \delta}$ the range of $\mathscr{W}_{f^{m}(x)}^{\mathrm{s}}$,

3) $T_{f^{m}(x)} W_{f^{m}(x)}^{\mathrm{s}, \delta}=E_{f^{m}(x)}^{\mathrm{s}}$.

4) $f\left(W_{f^{m}(x)}^{\mathrm{s}, \delta}\right) \subset W_{f^{m+1}(x)}^{\mathrm{s}, \delta}$.

5) There are numbers $\delta>0, C>0, \varepsilon>0$ independent of the point $x \in \Omega_{\varepsilon}$ such that:

$$
\begin{aligned}
y \in W_{x}^{\mathrm{s}, \delta} & \Leftrightarrow \lim _{N \rightarrow \infty} \frac{1}{N} \log d\left(f^{N}(x), f^{N}(y)\right)<0 \text { and } d(x, y) \leqq \delta \\
& \Leftrightarrow d\left(f^{N}(x), f^{N}(y)\right) \leqq C \exp \left(\gamma_{-}+\varepsilon\right) d(x, y) \text { and } d(x, y) \leqq \delta .
\end{aligned}
$$

6) The mapping defined on $\Omega_{\varepsilon}$ by $x \mapsto \mathscr{W}_{x}^{\mathrm{s}}$ is continuous when the curves are given the $C^{k-1}$ topology.

Remark. $W_{x}^{\mathrm{s}, \delta}$ is usually called the local stable manifold to distinguish it from the global stable manifold $W_{x}^{\mathrm{s}}=\bigcup_{n=1}^{\infty} f^{-n}\left(W_{f^{n}(x)}^{\mathrm{s}}\right) . \mathscr{W}_{x}^{\mathrm{s}}$ is a parametrization of the stable manifold and is highly non-unique.

Proof. The proof - except for 6) - can be obtained by reading carefully most of the statements of the stable manifold theorem. One only has to observe that the size of the $C^{k}$ norms only depends on the constants which enter in the definition of hyperbolicity for one orbit. By the construction of $\Omega_{\varepsilon}$, these constants are uniform for all $x \in \Omega_{\varepsilon}$.

The statement we know which makes most clear the dependence on the constants is that of [FHY]. One niggling point, however, is that due to the method of proof of [FHY] - using Irwin's method - the authors of this paper only obtain that the $W^{\mathrm{s}}$ are $C^{k-\varepsilon}$. This loss of $\varepsilon$ derivatives does not belong and the details are 
worked out in other references such as [PS2]. The loss of $\varepsilon$ derivatives will make no difference in the arguments that follow.

The proof of 6) can be obtained by reading carefully the proofs in [HPS]. This will allow to show that the mapping $x \mapsto \mathscr{W}_{x}^{\mathrm{s}}$ with the curves given the $C^{k-1}$ topology is as differentiable as the mapping $x \mapsto E_{x}^{\mathrm{s}}$.

An alternative argument is to use the closed graph theorem. If we have a sequence $\left\{x_{n}\right\} \subset \Omega_{\varepsilon}$ with $\lim x_{n}=x \in \Omega_{\varepsilon}$, due to the uniform bounds in 1), we can extract a subsequence $x_{n_{i}}$ in such a way that $\mathscr{W}_{x_{n_{1}}}^{\mathrm{s}}$ converges in the $C^{k-1}$ topology to a cetain $\tilde{\mathscr{W}}_{x}$. Using the characterization of the stable manifold in 5 ), we can conclude that $\tilde{\mathscr{W}}_{x}=\mathscr{W}_{x}^{\mathrm{s}}$. It is an easy proposition in point set topology that when all subsequences of a subsequence have the same limit and there is one convergent subsequence, then the full sequence has a limit.

Notation. If $\Omega$ is a set, we denote by

$$
W_{\Omega}^{\mathrm{s}, \delta}=\bigcup_{x \in \Omega} W_{x}^{\mathrm{s}, \delta} ; \quad W_{\Omega}^{\mathrm{u}, \delta}=\bigcup_{x \in \Omega} W_{x}^{\mathrm{u}, \delta}
$$

As a corollary of the stable manifold theorem we have some sort of "local product structure."

Corollary 5.3. Let $x \in \Omega_{\varepsilon}$ as before. For every $y \in \Omega_{\varepsilon}, d(x, y) \leqq K_{\Omega_{\varepsilon}}$ we have

$$
W_{y}^{\mathrm{s}, \delta} \cap W_{x}^{\mathrm{u}, \delta} \neq \emptyset, \quad W^{\mathrm{s}, \delta} \cap W_{y}^{\mathrm{u}, \delta} \neq \emptyset .
$$

Moreover, we can bound the length of the segments of $W_{x}^{\mathrm{s}}$ joining $x$ to $\left(W_{x}^{\mathrm{s}, \delta} \cap W_{y}^{\mathrm{u}, \delta}\right)$ and of $W_{x}^{\mathrm{u}}$ joining $x$ to $\left(W_{x}^{\mathrm{u}, \delta} \cap W_{y}^{\mathrm{s}, \delta}\right)$ by a constant times the distance of $x$ to $y$.

Remark. Notice that one corollary of this theorem is that the length of the stable unstable manifolds of $x$ can be bounded uniformly from below when $x \in \Omega_{\varepsilon}$. The bound, nevertheless, could depend on $\varepsilon$.

If $\mu$ is an invariant measure and $f$ has non-zero positive Lyapounov exponents, Property 5) implies that the relation $x \approx y$, given by $x \in W_{y}^{\mathrm{s}}$, is an equivalence relation. The partition into leaves $W_{x}^{\text {s }}$ can fail to be a foliation because there can be a non-empty set (but of measure zero) for which $W_{x}^{\mathrm{s}}=\{x\}$. Since the definitions Definition 3.5 and Definition 3.6 use more measure theory than continuity, they make sense in this generality, provided that one considers all statements a.e. $\mu$.

The following is a particular case of theorems proved in [LS, LY1].

Theorem 5.4. Let $f$ be $a C^{k}, k=2,3, \ldots, \infty$ diffeomorphism preserving an absolutely continuous measure $\mu$ and having no zero Lyapounov exponent with respect to $\mu$.

1) There exist expanding partitions adapted to $W^{\mathrm{u}}$.

2) For every expanding partition, the conditional measures $\mu_{x}^{\xi_{N}}$ are absolutely continuous with respect to the Lebesgue measure induced by the metric on $W_{x}^{\mathrm{s}}$.

3) Equation (3.3) holds a.e. $\mu$.

Proof. The existence of increasing partitions is proved in [LY1], p. $513 \mathrm{ff}$. The fact that (3.3) holds in Lemma 6.1 of [LY1] and 2) can be read off from the arguments in this paper. Actually this paper is the proof of the necessity and sufficiency of condition 2) for the validity of Pesin's entropy formula, originally proved for absolutely continuous invariant measures. 
Now' we can complete the proof of Theorem 1.3 under hypothesis i). By the absolute continuity properties of $h, \mu^{f}, \mu^{g}$ and the ergodicity, we have that $h_{*} \mu^{f}=\mu^{g}$. Since $h$ is continuous, $h\left(W_{x}^{\delta(f)}\right)=W_{h(x)}^{\delta^{\prime}(g)}$. As in the previous section, we observe that the restriction of $\mu^{f}$ to $W_{x}^{\mathrm{u}} \delta(f)$ is pushed by $h$ into the restriction of $\mu^{g}$ to $W_{x}^{\mathrm{u} \delta^{\prime}(g)}$.

By the same argument as in the previous section, we can conclude that (4.2) is valid. The following lemma states precisely the differentiability properties of the functions appearing in (4.2).

Lemma 5.5. The functions $\Delta_{x}^{\mathrm{u}}(y), \Delta_{x}^{\mathrm{s}}(y)$ satisfying (3.3), can be defined for all $x \in \Omega_{\varepsilon}$ and $y \in W_{x}^{\mathrm{s}, \delta}$. Moreover, if the diffeomorphism is $C^{k}$, they are $C^{k-1}$ and can be bounded uniformly in $C^{k-1}, k=2,3, \ldots$ provided that $\varepsilon, \delta$ are small enough.

Remark. Contrary to what happened in the case of uniform hyperbolicity, the smallness conditions in $\varepsilon, \delta$ depend on $k$. So that even for a $C^{\infty} f$, we cannot conclude a $C^{\infty}$ analogue of our theorem.

Proof. As in Corollary 4.4, we can write

$$
\ln \left(\Psi_{-n}^{\mathrm{u}}(x)\right)-\ln \left(\Psi_{-n}^{\mathrm{u}}(y)\right)=\sum_{i=0}^{n-1}\left[\ln \Psi_{-1}^{\mathrm{u}}\left(f^{-i}(x)\right)-\ln \Psi_{-1}^{\mathrm{u}}\left(f^{-i}(y)\right)\right],
$$

and we want to bound the supremum of the first $k-1$ derivatives with respect to $y$ of each of the terms in the sum by an absolutely convergent series, which we can choose uniformly in $x \in \Omega_{\varepsilon}$.

We claim that

$$
\sup _{y \in W_{x}^{\mathrm{u}, \delta}}\left|D_{\mathrm{u}}^{r} \Psi_{-1}^{\mathrm{u}}\left(f^{-n}(y)\right)\right| \leqq K \exp (-\gamma n) \exp (\varepsilon r n) r n^{r}
$$

where $K$ can be chosen uniformly for all $x \in \Omega_{\varepsilon}$.

In effect, we observe that if we take $r$ derivatives and use the chain rule and the rule for the derivative of products as often as possible, we obtain that the derivatives we are bounding can be written as the sum of $N(r, n)$ terms, each of them having not more than $F(r, n)$ elementary factors. Each elementary factor is a derivative of $\Psi_{-1}^{\mathrm{u}}$ or of $f^{-1}$ evaluated at $f^{-i}(y), i \leqq n$. The rule for products gives that $N(r+1, n) \leqq N(r, n) F(r, n)$ and by the chain rule, $F(r+1, n) \leqq F(r, n)+n$, so that $F(r, n) \leqq r n, N(r, n) \leqq n^{r} r !$ factors:

We furthermore observe that if we call a "segment" a product of consecutive

$$
S(i, j) \equiv D f^{-1}\left(f^{-i}(y)\right) D f^{-1}\left(f^{-i-1}(y)\right) \ldots D f^{-1}\left(f^{-j}(y)\right),
$$

each term consists of not more than $r$ segments and $r$ derivatives of order not higher than $r$.

By 1) of Theorem 5.1 we can bound on $W_{x}^{\mathrm{u}}, x \in \Omega_{\varepsilon} \sup \|S(i, j)\| \leqq \exp (-(i-j) \gamma$ $+\varepsilon j)$. We furthermore observe that the total length of all the segments is at least $n-r+1$ since a segment containing segments of total length $l$, when we take derivatives, produces segments which have length at least $n-1$.

Using the triangle inequality, we have produced the bounds we claimed in (5.1). This finishes the proof of Lemma 5.5. The same argument can be used to finish the proof Lemma 4.3.

For the case of uniformly hyperbolic systems, we can perform the same argument but take $\varepsilon \equiv 0$ in the estimates. This establishes Lemma 4.3 except for the 
claim about the uniform modulus of continuity, which we now proceed to prove.

If $\rho(t)$ is a modulus of continuity of $D^{k-1} \Psi_{-1}^{\mathrm{u}}(y)$, that is:

$$
\sup _{d\left(y, y^{\prime}\right) \leqq t}\left|D_{\mathrm{u}}^{k-1} \Psi_{-1}^{\mathrm{u}}(y) D_{\mathrm{u}}^{k-1} \Psi_{-1}^{\mathrm{u}}\left(y^{\prime}\right)\right| \leqq \rho(t),
$$

then, we can bound $\sup _{d\left(y, y^{\prime}\right) \leqq t}\left|D_{\mathrm{u}}^{k-1} \Delta^{\mathrm{u}}(y)-D_{\mathrm{u}}^{k-1} \Delta^{\mathrm{u}}\left(y^{\prime}\right)\right|$ by summing the bounds for each of the terms in the sum defining $\Delta$ in (3.3).

We observe that in the expression of $D_{\mathrm{u}}^{k-1} \Psi_{-1}^{\mathrm{u}}\left(f^{-n} y\right)$ obtained by applying the chain rule and the rule for derivatives of the product as often as possible there is one term which is $\left[D_{\mathrm{u}}^{k-1} \Psi_{-1}^{\mathrm{u}}\right]\left(f^{-n} y\right) D f^{-n}(y)^{\otimes n}$, and all the others involve derivatives of order strictly smaller than $k-1$. This first term has a modulus of continuity $\rho(t) \lambda^{n}$.

Each one of the terms involving derivatives of order less than $k-1$ will have a modulus of continuity $\rho^{\prime}(t)=r\left(\sup _{r \leqq k-1} \max \left\{\left\|D^{r} \Psi\right\|, D^{r} f^{-1}(y)\right\}\right)^{k-1} \lambda^{n-k}|t|$ as can be shown using the facts that each of the functions appearing as factors in a term are Lipschitz, the product rule for Lipschitz constants and that the Lipschitz constant can be bounded by the supremum of the norm of the derivative.

Since we have shown that there are at most $r ! n^{r}$ of those terms, the modulus of continuity can be summed in $n$ uniformly for all the points claimed. This finishes the proof of Lemma 4.3.

The same argument used in the proof of Lemma 4.5 allows us to conclude

Lemma 5.6. Under the assumptions of Theorem 1.3, if $x \in \Omega_{\varepsilon}$ as in Theorem 5.1, then provided that $\delta$ is small enough, $h \backslash_{W_{x}^{\mathrm{s} s}}$ is $C^{k}, k=1, \ldots$ and, moreover, we can bound uniformly $\left\|J^{k}\left(h \backslash_{W_{x}^{\text {s. } \delta}}, M\right)\right\|$.

Notation. We will denote by

$$
\|\Phi\|_{C_{\mathrm{s}}^{N}}=\sup _{x \in \Omega_{\varepsilon}}\left\|\Phi \circ W_{x}^{\mathrm{s}, \delta}\right\|_{C^{N}}
$$

and similarly for $C_{\mathrm{u}}^{N}$ or for Hölder spaces.

The only ingredient we need to conclude the proof of Theorem 1.3 is an analogue of Lemma 3.2. The main complication is that we only have control of the regularity on $\bigcup_{x \in \Omega_{\varepsilon}} W_{x}^{\mathrm{u}, \delta} \cup W_{x}^{\mathrm{u}, \delta}$, a set which has a complicated geometric structure.

The following is the main technical lemma that we will prove by a strategy inspired by that of [Jo2]:

Theorem 5.7. Let $\Omega$ be a subset of $\mathbf{R}^{n}$ and $k$ be a natural number such that through every point $x \in \Omega$ we can find two functions: $\mathscr{W}_{x}^{\mathrm{s}}$, (respectively $\left.\mathscr{W}_{x}^{\mathrm{u}}\right):[-1,1]^{\mathrm{u}}$ (respectively $\left.[-1,1]^{s}\right) \mapsto \mathbf{R}^{n}$ with the following properties:

i) $\mathscr{W}_{x}^{\mathrm{s}}, \mathscr{W}_{x}^{\mathrm{u}}$ are uniformly $C^{k}$.

ii) $x \mapsto \mathscr{W}_{x}^{\mathrm{s}}, x \mapsto \mathscr{W}_{x}^{\mathrm{u}}$ are continuous when the mappings are given the $C^{k}$ topology.

iii) $T_{x} W_{x}^{\mathrm{u}, \delta}$ is transversal to $T_{x} W_{x}^{\mathrm{s}, \delta}$ and the angle is bounded from below uniformly in $x$.

Assume that $x$ is a point in $\Omega$ such that we can find a sequence $\left\{x_{n}\right\} \subset \Omega$, $\lim x_{n}=x$ satisfying for some $\gamma^{\prime}<\gamma<1$ and some $\beta>0$, 
iv) $(\gamma)^{-1} \leqq \frac{d\left(W_{x_{n}}^{\mathrm{s}, \delta} \cap W_{x}^{\mathrm{u}, \delta}, W_{x_{n+1}}^{\mathrm{s}, \delta} \cap W_{x}^{\mathrm{u}, \delta}\right)}{d\left(W_{x_{n+1}}^{\mathrm{s}, \delta} \cap W_{x}^{\mathrm{u}, \delta}, W_{x_{n+2}}^{\mathrm{s}, \delta} \cap W_{x}^{\mathrm{u}, \delta}\right)} \leqq\left(\gamma^{\prime}\right)^{-1}$,

$$
\begin{gathered}
(\gamma)^{-1} \leqq \frac{d\left(W_{x_{n}}^{\mathrm{u}, \delta} \cap W_{x}^{\mathrm{s}, \delta}, W_{x_{n+1}}^{\mathrm{u}, \delta} \cap W_{x}^{\mathrm{s}, \delta}\right)}{d\left(W_{x_{n+1}}^{\mathrm{u}, \delta} \cap W_{x}^{\mathrm{s}, \delta}, W_{x_{n+2}}^{\mathrm{u}, \delta} \cap W_{x}^{\mathrm{s}, \delta}\right)} \leqq\left(\gamma^{\prime}\right)^{-1}, \\
d\left(x, x_{0}\right) \leqq \beta .
\end{gathered}
$$

Let $\phi$ be a continuous function $\Omega \mapsto \mathbf{R}$ satisfying:

v) For some $A \in \mathbf{R}^{+}\left\|\phi \circ \mathscr{W}_{x}^{\mathrm{s}, \delta}\right\|_{C^{k}} \leqq A,\left\|\phi \circ \mathscr{W}_{x}^{\mathbf{u}, \delta}\right\|_{C^{k}} \leqq A$ uniformly in $x \in \Omega$.

vi) Let $k^{\prime}$ be such that $k^{\prime} \leqq k$,

$$
\left(\gamma / \gamma^{\prime}\right)^{2 k^{\prime}} \gamma^{1 / 2}<1, \quad \gamma^{1 / 2}\left(\gamma / \gamma^{\prime}\right)^{2 k^{\prime}}<1
$$

1) We can find a polynomial $P_{x}^{k^{\prime}}$ of order $k^{\prime}$ in such a way that

$$
\left|\phi(y)-P_{x}^{k^{\prime}}(y)\right| \leqq K_{1}|x-y|^{k^{\prime}}
$$

whenever $y \in W_{\Omega}^{\mathrm{s}, \delta} \cup W_{\Omega}^{\mathrm{u}, \delta} d(x, y) \leqq \beta$.

2) $\left\|P_{x}^{k^{\prime}}\right\|_{C^{k^{\prime}}} \leqq K_{2} A$, where $K_{2}$ is a constant that depends only on $k, \varepsilon, \gamma, \gamma^{\prime}, \beta$ and the uniform bounds assumed in i)-vi).

3) If $\Lambda$ is a subset of $\Omega$ where the constants $\gamma, \gamma^{\prime}$ and $\beta$ can be chosen uniformly, the mapping $x \mapsto P_{x}^{k}$ is continuous when restricted to $\Lambda$.

Remark. We emphasize that given $k^{\prime}<k$, assumption iv) of Theorem 5.7 will be verified if $\gamma, \gamma^{\prime}$ are close enough.

Proof. We will prove first the existence of $P_{x}^{n}$ for a fixed $x$.

To simplify the notation, we will omit the subindex $x$ for $P$ till we start discussing continuity with respect to $x$. Since differences with the point at which we are approximating appear frequently, we will change coordinates to that this point is the origin. We will also assume that the point at which we are approximating is the origin.

Following [Jo2], we will assume that the dimensions of the stable and unstable manifolds are 1 so as to avoid notational complications. Moreover, this is the only case of the theorem we are going to use in this paper.

Since the stable and unstable manifolds of $x$ are $C^{k}$, we can perform a $C^{k}$ change of variables so that they become the coordinate axes. Moreover, since the stable and unstable manifold satisfy uniform $C^{k}$ estimates, the fact that we will have to choose different coordinates systems for different points will not change the uniform estimates we claim.

The proof of this result will follow the general strategy of [Jo2]: we determine $P^{N}$ as the limit of polynomials obtained by interpolation on grids which are approximately of the form $\left\{\left(r^{i}, r^{j}\right)\right\}_{i, j=N}^{N+n}$ and truncating the terms of high order. Our choice of grids has to be different from that of [ $\mathrm{Jo} 2]$ because we have control of the function on a set with complicated geometry. This also complicates some of the following arguments.

We will proceed by induction so that we will assume that $P^{N}$ exists (satisfying property 2) with $k^{\prime}=N$ and will conclude that $P^{N+1}$ exists and that it satisfies property 2) with $k^{\prime}=N+1$. Moreover, by considering $\phi(x)-P^{N}(x)$, we can assume inductively that $|\phi(x)| \leqq K|x|^{N-\varepsilon}$.

Call $\mathscr{P}_{i j}=\mathscr{W}_{x_{i}}^{\mathrm{u}, \delta} \cap \mathscr{W}_{x_{j}}^{\mathrm{s}, \delta}$, and we will denote the coordinates of these points by $\mathscr{P}_{i j}^{1}$ and $\mathscr{P}_{i j}^{2}$ respectively. We will also call $\mathscr{G}\left(N, M ; N^{\prime}, M^{\prime}\right)=\left\{\mathscr{P}_{i j} \mid i=\right.$ 
$\left.M, \ldots, M+N, j=M^{\prime}, M^{\prime}+N^{\prime}\right\}$, where $\left\{x_{i}\right\}_{n=0}^{\infty}$ is the sequence of points we assumed in iv). When $N=N^{\prime}$ and $M=M^{\prime}$ we will simply write $\mathscr{G}(N, M)$.

The following proposition is a quantitative version of the well known Lagrange interpolation:

Proposition 5.8. Given a grid of the form $\left\{\left(x_{i}^{1}, x_{j}^{2}\right), i, j=0, N\right\}$ and a collection of $(N+1)^{2}$ numbers $a_{i j}$ we can find a unique polynomial $P$ of degree $N$ on each variable in such a way that

$$
P\left(x_{i}^{1}, x_{j}^{2}\right)=a_{i j}, \quad i, j=N, N+M
$$

Moreover,

$$
\begin{aligned}
\|P\|_{l^{1}, R} & \leqq\|a\| N^{2}\left(\frac{\sup _{i}\left(R+\left|x_{i}^{1}\right|\right)}{\inf _{\substack{i, i^{\prime} \\
i \neq i^{\prime}}}\left|x_{i}^{1}-x_{i^{\prime}}^{1}\right|}\right)^{N}\left(\frac{\sup _{j}\left(R+\left|x_{j}^{2}\right|\right)}{\inf _{\substack{j, j^{\prime} \\
j \neq j^{\prime}}}\left|x_{j}^{2}-x_{j^{\prime}}^{2}\right|}\right)^{N} \\
& \equiv\|a\| K\left(\left\{x_{i}^{1}\right\},\left\{x_{j}^{2}\right\} ; R\right),
\end{aligned}
$$

where $\|P\|_{l^{1}, R}=\sum\left|P_{k}\right| R^{|k|}$ is a weighted $l^{1}$ norm. $\|a\|$ is the supremum of the absolute values of the terms in the sequence.

Proof. Recall the Lagrange formula for the interpolating polynomial:

$$
P\left(x^{1}, x^{2}\right)=\sum_{i j} a_{i j} \frac{\prod_{i^{\prime} j^{\prime}}\left(x^{1}-x_{i^{\prime}}^{1}\right)\left(x^{2}-x_{i^{\prime}}^{2}\right)}{\prod_{\substack{i^{\prime} \neq \neq j \\ j_{j}^{\prime}}}^{2}\left(x_{i}^{1}-x_{i^{\prime}}^{1}\right)\left(x_{j}^{2}-x_{j^{\prime}}^{2}\right)} .
$$

Clearly, $\left\|x^{1}-x_{i^{\prime}}^{1}\right\|_{l^{1}, R}=R+\left|x_{i}^{1}\right|$ and similarly for the factors with $x^{2}$. Recall that, \|\|$_{l^{1}, R}$ satisfies the Banach algebra property: $\|P Q\|_{l^{1}, R} \leqq\|P\|_{l^{1}, R}\|Q\|_{l^{1}, R}$.

Remark. For two dimensional interpolation grids which are cartesian products of one dimensional grids, it is useful to think of the Lagrange formula as interpolating a polynomial in $x^{1}$ taking values in the polynomials in $x^{2}$. The polynomials in $x^{2}$ are obtained by interpolating on the vertical lines.

Remark. Notice that if we take $R=\sup _{i, j}\left\{\left|x_{i}^{1}\right|,\left|x_{j}^{2}\right|\right\}$. Equation (5.3) becomes $\|P\|_{l^{1}, R} \leqq\left\|a_{i j}\right\| K$, where $K$ remains bounded if the ratios of the coordinates of the points in the grid remain bounded even if the coordinates become close to zero. This will be important when we consider the limit as the interpolating grid converges to the origin.

The following is a version of Lemma 1 of [ $\mathrm{Jo} 2]$ that tells us that if a grid is not exactly a product grid but is close enough to be a product grid - the grids for which the Lagrange interpolation applies - we can still interpolate and obtain an interpolating polynomial which is uniquely defined.

Lemma 5.9. Let $\mathscr{G}^{0}=\left\{\left(x_{i}^{1}, x_{j}^{2}\right) \mid i, j=0, N\right\}$ be a product grid such as those considered in Proposition 5.8. Let $\mathscr{G}=\left\{p_{i j} \mid i, j=0, N\right\}$ be another grid of points such that: $\Gamma \equiv \sup \left|p_{i j}-\left(x_{i}^{1}, x_{j}^{2}\right)\right| \leqq \frac{1}{2 N^{2} K\left(\left\{x_{i}^{1}\right\},\left\{x_{j}^{2}\right\}\right)} \sup \left(\left|p_{i j}\right|,\left|\left(x_{i}^{1}, x_{j}^{2}\right)\right|\right)$. Then, given $(N+1)^{2}$ numbers $a_{i j}$ it is possible to find a polynomial $P$ such that $P\left(p_{i j}\right)=a_{i j}$, and in such a way that:

$$
\|P\| \leqq\|a\|_{l^{1}} K^{\prime},
$$

where $K^{\prime}=\left(1+\frac{\Gamma N^{2} K}{1-\Gamma N^{2} K}\right)$. 
Remark. Notice that, unfortunately, the conditions we have to impose depend on $N$, the degree of the polynomial. This will make the discussion of a $C^{\infty}$ version of Theorem 1.3 somewhat complicated.

Proof. Notice that if $P^{1}$ is the polynomial obtained interpolating on the grid $\mathscr{G}^{0}$ rather that on the grid $\mathscr{G}$, we would have $\left|P^{1}\left(p_{i j}\right)-a_{i j}\right| \leqq \Gamma \sup \left\|D P^{1}\right\|$. For any polynomial of degree $N$, we have $\sup \|D P\| \leqq\|P\|_{l^{1}} N^{2}$. Substituting (5.3) in the last inequalities, we obtain:

$$
\left|P^{1}\left(p_{i j}\right)-a_{i j}\right| \leqq \Gamma N^{2} K\|a\| .
$$

We can now consider the polynomial $P^{2}$ which on the grid $\mathscr{G}^{0}$ takes the values $a_{i j}-P^{1}\left(p_{i j}\right)$.

The previous argument shows that $\left|P^{1}\left(p_{i j}\right)+P^{2}\left(p_{i j}\right)-a_{i j}\right| \leqq\left(\Gamma N^{2} K\right)^{2}\|a\|$.

If we construct a sequence of polynomials $P^{1}, P^{2}, \ldots, P^{n}, \ldots$ such that $P^{n}$ interpolates on $\mathscr{G}^{0}$ the values $a_{i j}-P^{1}\left(p_{i j}\right)-P^{2}\left(p_{i j}\right)-\cdots-P^{n-1}\left(p_{i j}\right)$, we will have

$$
\left|P^{1}\left(p_{i j}\right)+P^{2}\left(p_{i j}\right)+\cdots+P^{n}\left(p_{i j}\right)-a_{i j}\right| \leqq\left(\Gamma N^{2} K\right)^{n}\|a\| .
$$

Hence, we can prove inductively that $\left\|P^{n}\right\|_{l^{1}} \leqq\left(\Gamma N^{2} K\right)^{n}\|a\|$.

We now continue with the proof of Theorem 5.7.

We observe that the conditions i) of Lemma 5.9 are scale invariant. They depend only on relative ratios of the distances to the origin of the points in the grid. This scale invariance and the continuity of the stable and unstable manifolds with respect to the base point when the base point ranges in $\Omega_{\varepsilon}$ shows that the conditions of this lemma will be satisfied for all the grids $\mathscr{G}\left(N, M ; N^{\prime}, M^{\prime}\right)$ provided that we assume that $\beta$ is small enough. (How small depends on $N$ as well as on the modulus of continuity of the foliations.) Therefore, if smallness assumptions on $\beta$ in Theorem 5.7 hold (we will later on show that all the smallness assumptions can be met by choosing constants in an appropriate order), we can construct polynomials of degree $N$ on each variable and which agree with $\Phi$ on a grid.

We want to show that, as we shrink this grid to the origin, there is a well defined limit. We will estimate the changes when we shrink both coordinates by a factor and show that the changes can be bounded by a uniformly convergent sequence. Properties of the resulting limit will be established by looking carefully at the limiting process.

It suffices to obtain estimates of the effect of shrinking one of the coordinates. The same argument applied with the role of the stable and unstable reversed will establish similar estimates for the effect of shrinking the grid in the other coordinate. By applying these two steps in succession we can shrink both coordinates and the effect will be estimated by a uniformly convergent series.

If the grids we consider were exactly products, we could think of the interpolation on the grid as interpolating a $P_{\left(x_{1}\right)}^{1}$ polynomial in $x^{1}$ whose coefficients are polynomials in $x^{2}$. The interpolation is done by imposing $P^{1}\left(x_{i}^{1}\right)=P_{i}^{2}\left(x_{2}\right)$, where $P_{i}^{2}\left(x^{2}\right)$ is obtained by interpolating in $x_{2}$ the $P_{i}^{2}\left(x_{j}^{2}\right)=\phi\left(x_{i}^{1} x_{j}^{2}\right)$.

In view of the stability of Lagrange interpolation, to estimate the effect of changing the $x_{j}^{2}$ in the grid it suffices to control the changes on $P_{i}^{2}\left(x_{2}\right)$. Since the $x_{i}^{1}$ are kept constant in this argument, it is plausible that only the smoothness properties of the restrictions of $\phi$ to vertical lines matter. 
In our situation, the grids are not exactly products, but they are close enough that the argument goes through.

Proposition 5.10. If $P^{N, M ; N^{\prime}, M^{\prime}}$ denotes the polynomial obtained by interpolating the function $\phi$ using Proposition 5.8 or $\mathscr{G}\left(N, M ; N^{\prime}, M^{\prime}\right)$ then we have

$$
\begin{aligned}
& \left\|P^{N, M ; N^{\prime}, M^{\prime}}-P^{N, M+1 ; N, M+1}\right\|_{l^{1}, R} \\
& \quad \leqq C\|\phi\|_{C_{\mathrm{s}}^{N-2}}\left(\sup _{j=M \cdots N+M+1}\left|x_{j}^{2}-x_{N+M+1}^{2}\right|\right)^{N+2}+\varepsilon\left\|P^{N, M ; N, M+1}\right\|_{l^{1}, R},
\end{aligned}
$$

where $R=\sup \left\{\left|x_{i}^{1}\right|\left|x_{j}^{2}\right|\right\}$ and $C$ is a constant that depends on the ratios of the coordinates of the grid but not on the absoloute scale. $\varepsilon$ is a number that can be made arbitrarily small by choosing $\beta$ sufficiently small.

Proof. In view of Lemma 5.9 it suffices to show that:

$$
\sup _{\mathscr{R}_{i j} \in \mathscr{G}(N, M ; N, M+1)}\left|P^{N, M ; N, M}\left(\mathscr{P}_{i j}\right)-\phi\left(\mathscr{P}_{i j}\right)\right| \leqq\|\phi\|_{C_{\mathrm{s}}^{N+2}}+\varepsilon\left\|P^{N, M ; N, M}\right\|_{l^{1}, R} .
$$

Notice that, by consruction of $P^{N, M ; N, M}$, this difference is zero for $P_{i j} \in \mathscr{G}(N, M ; N, M)$ so that we only have to estimate the difference on the points in which the two grids differ. This is just the last row

$$
\left\{\mathscr{P}_{i, M^{\prime}+N+1}\right\}_{i=M}^{M+N} \text {. }
$$

If $W_{\left(x_{i}^{1}, 0\right)}^{\mathrm{s}, \delta}$ were a vertical line $\left(x_{i}^{1}, x^{2}\right)$ we could use the classical estimates for the error in Lagrange interpolation (see e.g. [SB] Theorem 2.1.4.1)

$$
\begin{aligned}
& \left|P^{M N ; N M}\left(\mathscr{P}_{i, M+N+1}\right)-\phi\left(\mathscr{P}_{i, M+N+1}\right)\right| \\
& \quad \leqq \frac{1}{(M+2) !}\|\phi\|_{C_{\mathrm{s}}^{N+2}}\left(\sup _{j=M, \ldots, N+M+1}\left|x_{j}^{2}-x_{N+M+1}^{2}\right|\right)^{N+2} .
\end{aligned}
$$

These are the estimates we want to establish with $\varepsilon=0$.

Notice also that it is posible to bound

$$
\sup _{|x| \leqq R}\left|D^{j} P\right| \leqq R^{-j}\|P\|_{l^{1}, R} K
$$

where $K$ depends only on the dimension.

Using Faa-di Bruno formula (see e.g., [AR] p. 3)

$$
\begin{aligned}
\left|D^{N+2} P \circ W_{(x)}\right| & =\sum_{1 \leqq q \leqq N+2} \sigma_{N+2, q} \sum_{i_{1} \ldots i_{q}=N+2}\left|D^{q} P \circ W_{(x)}\right| D^{i_{1}} W(x) \ldots D^{i q} W(x) \varepsilon^{q} \\
& \leqq \sum_{1 \leqq q \leqq N+2} \sigma_{N+2, q} \sum_{i_{1} \ldots i_{q}=N+2} R^{-q}\|P\|_{l^{1}, R} R^{q(N+2)-\sum i_{1} \ldots i_{q}} \\
& \leqq \varepsilon \sum_{1 \leqq q \leqq N+2} \sigma_{N+2, q}\|P\|_{l^{1}, R} R^{q(M+1)-q} \\
& \leqq \varepsilon\|P\|_{l^{1}, R} R^{N} .
\end{aligned}
$$

If we parametrize the stable manifold $W_{\left(x_{i}^{1}, 0\right)}^{s, \delta}$ by $x^{2}, P \circ W_{\left(x_{i}^{1}, 0\right)}^{s, \delta}$ will not be a polynomial but can be approximated by one up to errors which can be bounded by the $N+2$ derivative of $P \circ W_{\left(x_{i}^{1}, 0\right)}^{s}$. 
Since $W_{\left(x_{i}^{1}, 0\right)}^{s, \delta}$ converges in the $C^{N+2}$ topology to a constant, we should have

$$
\left|D^{i} W_{\left(x_{i}^{1}, 0\right)}^{\mathrm{s}, \delta}\left(x^{2}\right)\right| \leqq \varepsilon\left(x_{i}^{1}\right)\left|x^{2}\right|^{N+2-i} .
$$

Hence in the neighborhood where $\left|\left(x^{1}, x^{2}\right)\right| \leqq R$, this is bounded by $\varepsilon R^{N+2-i}$.

Expresseed in components, (5.6) can be written as

$$
\begin{aligned}
\left|P_{i j}^{N, M}-P_{i j}^{N, M+1}\right| \leqq & \left(R_{N, M+1}\right)^{N+2^{-\imath-J}}\left(\|\phi\|_{C_{\mathrm{s}}^{N+2}}+\|\phi\|_{C_{\mathrm{u}}^{N+2}}\right) k \\
& +\varepsilon \sum_{i^{\prime}, j^{\prime}}\left|P_{i^{\prime}, j^{\prime}}^{N M}\right|\left(R_{N, M+1}\right)^{i^{\prime}+j^{\prime}-i-j} .
\end{aligned}
$$

Remark. Notice that in [Jo2] the bound that is used is slightly weaker since the fact that the leaves are converging to the axis is not used.

We also note an easy Corollary of (5.3)

Proposition 5.11. If $|\phi(x)| \leqq K|x|^{N}$ as in Theorem 5.7 then

$$
\left\|P^{N, M}\right\|_{l^{1}, R_{N, M}} \leqq K\left(R^{N, M}\right)^{N} .
$$

Hence,

$$
\left|P_{i j}^{N, M}\right| \leqq K\left(R^{N, M}\right)^{N-i-j} .
$$

Since we had $\beta\left(\gamma^{\prime}\right)^{M} \leqq R_{N, M} \leqq \beta \gamma^{M}$ we have

$$
\begin{aligned}
\left|P_{i j}^{N, M}-P_{i j}^{N, M+1}\right| \leqq & \gamma^{(M+1)(N+2-i-j)} K\left(\|\phi\|_{C_{\mathrm{s}}^{N+2}}+\|\phi\|_{C_{\mathrm{u}}^{N+2}}\right) \\
& +\varepsilon \sum_{i^{\prime}+j^{\prime} \leqq i+j} P_{i^{\prime}, j^{\prime}}^{N M} \gamma^{(M+1)\left(i^{\prime}+j^{\prime}-i-j\right)} \\
& +\varepsilon \sum_{i^{\prime}+j^{\prime}>i+j} P_{i^{\prime}, j^{\prime}}^{N M}\left(\gamma^{\prime}\right)^{(M+1)\left(i^{\prime}+j^{\prime}-i-j\right)}
\end{aligned}
$$

if $i+j \leqq N+2$

$$
\begin{aligned}
\left|P_{i j}^{N, M}-P_{i j}^{N, M+1}\right| \leqq & \left(\gamma^{\prime}\right)^{(M+1)(N+2-i-i)} K\left(\|\phi\|_{C_{\mathbf{s}}^{N+2}}+\|\phi\|_{\left.C_{\mathrm{u}}^{N+2}\right)}\right. \\
& +\varepsilon \sum_{i^{\prime}+j^{\prime} \leqq i+j} P_{i^{\prime}, j^{\prime}}^{N M} \gamma^{(M+1)\left(i^{\prime}+j^{\prime}-i-j\right)} \\
& +\varepsilon \sum_{i^{\prime}+j^{\prime}>i+j} P_{i^{\prime}, j^{\prime}}^{N M}\left(\gamma^{\prime}\right)^{(M+1)\left(i^{\prime}+j^{\prime}-i-j\right)}
\end{aligned}
$$

if $i+j>N+2$.

Where, as before $K$ depends only on the foliations and $\Omega$ and $\varepsilon$ can be made arbitrarily small by choosing a sufficiently small neighborhood.

From that, we claim that it follows by induction that:

$$
\left|P_{i j}^{N M}\right| \leqq \gamma^{(N+2-i-j) M} K\left(\|\phi\|_{C_{\mathrm{s}}^{N+2}}+\|\phi\|_{C_{\mathrm{u}}^{N+2}}\right)
$$

if $i+j>N+2$

$$
\begin{aligned}
\left|P_{i j}^{N, M}\right| \leqq & K\left(\|\phi\|_{C_{\mathrm{s}}^{N+2}}+\|\phi\|_{C_{\mathrm{u}}^{N+2}}\right) \\
& +\left[\sum_{m=0}^{M} \gamma^{(N+2-i-j) m}+\varepsilon \sum_{m=0}^{M} \sum_{i^{\prime} j^{\prime}}\left[\left(\gamma^{\prime}\right)^{N+2-i^{\prime}-j^{\prime}} \gamma^{i^{\prime}+j^{\prime}-i-j}\right]^{m}\right]
\end{aligned}
$$

if $i+j \leqq N+2$. 
Remark. Notice that, by assumption iii) in Theorem 5.7 we have that

$$
\begin{aligned}
\left(\gamma^{\prime}\right)^{N+2-i^{\prime}-j^{\prime}} \gamma^{i^{\prime}+j^{\prime}-i-j} & \leqq \gamma^{N+2-i-j}\left(\frac{\gamma}{\gamma^{\prime}}\right)^{N+2-i^{\prime}-j^{\prime}} \\
& \leqq \gamma^{N+2-i-j}\left(\frac{\gamma}{\gamma^{\prime}}\right)^{N+2} \leqq \gamma^{N+2-i-j-1 / 2}
\end{aligned}
$$

Hence, (5.12) implies that all coefficients $P_{i j}^{N, M}$ with $i+j \leqq N+1<N+3 / 2$ remain bounded (and, anon will show converge) and that $P_{i j}^{\bar{N}}, M \approx \gamma^{-1 / 2 M}$ when $i+j=N+2$. Similarly, when $i+j<N+2$

$$
P_{i j}^{N, M} \leqq K \gamma^{(N+3 / 2-i-j) M} .
$$

The induction claim (5.12) can be established very easily by observing that (5.12) claims that the $P_{i j}^{N M}$ grow as sums of exponentials.

If the growth were as claimed, the R.H.S. of (5.11) could also be bounded by exponentials. We only have to check that under (5.12), the dominant exponentials in the R.H.S. of (5.11) are such that they reproduced the desired result.

Notice that the bounds we have claimed for $\left|P_{i j}^{N M}\right|$ are really bounds for $\sum_{m=0}^{M}\left|P_{i j}^{N, M+1}-P_{i j}^{N, M}\right|$ so that in the cases that these bounds are uniform in $M$, we have really established that $P_{i j}^{N, M}$ has a limit as $M \rightarrow \infty$.

We claim that the polynomials $P^{N}$ in Theorem 5.7 can be constructed as $P_{i j}^{N}=\lim _{M \rightarrow \infty} P_{i j}^{N, M}$ if $i+j \leqq N+1, P_{i j}^{N}=0$ otherwise.

We start by estimating the difference with the approximating polynomials. We want to establish that

$$
\sup _{|x| \leqq R^{N, M}}\left|P_{(x)}^{N}-P_{(x)}^{N, M}\right| \leqq K\left(R^{N, M}\right)^{N+3 / 2} .
$$

Observe that:

$$
\begin{aligned}
\sup _{|x| \leqq R^{N, M}}\left|P_{(x)}^{N}-P_{(x)}^{N, M}\right| \leqq & \sum_{i+j \leqq N+1} \sum_{m=M}^{\infty}\left|P_{i j}^{N, M}-P_{i j}^{N, M+1}\right|\left(R^{N, M}\right)^{i+j} \\
& +\sum_{i+j=N+2}\left|P_{i j}^{N, M}\right|\left(R^{N, M}\right)^{i+j} \\
& +\sum_{i+j>N+2}\left|P_{i j}^{N, M}\right|\left(R^{N, M}\right)^{i+j}
\end{aligned}
$$

We want to establish that each of these terms can be bounded by a constant times $\left(R^{N, M}\right)^{N+1+1 / 2}$. Since $R^{N, M} \geqq \beta \gamma^{\prime M}$ it suffices bound by $\gamma^{(N+2+1 / 2) M}$.

By (5.12) we have (up to constant, which we will not write)

$$
\begin{aligned}
\sum_{i+j>N+2}\left|P_{i j}^{N, M}\right|\left(R^{N, M}\right)^{i+j} & \leqq \sum_{i+j>N+2} \gamma^{\prime(N+2-i-j) M} \gamma^{M\left(i_{j}\right)} \\
& =\gamma^{\prime(N+3 / 2) M} \sum_{i+j}\left[\gamma^{\prime 1 / 2}\left(\frac{\gamma}{\gamma^{\prime}}\right)^{i+j}\right]^{M} .
\end{aligned}
$$

Under hypothesis v) of Theorem 5.7,

$$
\gamma^{\prime 1 / 2}\left(\frac{\gamma}{\gamma^{\prime}}\right)^{i+j} \leqq \gamma^{\prime 1 / 2}\left(\frac{\gamma}{\gamma^{\prime}}\right)^{2 N}<1
$$


Hence, this term can be bounded in the desired form.

Using (5.12) and the remark immediately after, we have

$$
\sum_{i+j=N+2}\left|P_{i j}\right|\left(R^{N, M}\right)^{i+j} \leqq \gamma^{-1 / 2 M}\left(R^{N, M}\right)^{N+2} .
$$

It suffices to observe that $\left(R^{N M}\right)^{1 / 2} \gamma^{-1 / 2}$ is bounded by a constant.

If $i+j<M+1$ we observe that by (5.9) we have

$$
\left|P_{i j}^{N, m}-P_{i j}^{N, m+1}\right|\left(R^{N, m}\right)^{i+j} \leqq\left[\left(R^{N, m}\right)^{N+2}+\varepsilon \sum_{i^{\prime}, j^{\prime}}\left|P_{i^{\prime} j^{\prime}}^{N, M}\right|\left(R_{N, m}\right)^{i^{\prime}+j^{\prime}}\right] K .
$$

Using (5.12) we furthermore have

$$
\begin{aligned}
\sum_{i^{\prime} j}\left|P_{i^{\prime}, j^{\prime}}^{N M}\right|\left(R_{N m}\right)^{i+j} \leqq & K \sum_{i^{\prime}+j^{\prime}>N+2} \gamma^{\left(N+2-i^{\prime}-j^{\prime}\right) M} \gamma^{\left(i^{\prime}+j^{\prime}\right) M} \\
& +\sum_{i^{\prime}+j^{\prime}=N+2} \gamma^{[N+2-1 / 2] m}+\sum_{i+j<N+2} \gamma^{(N+3 / 2-1) m} \\
\leqq & K \gamma^{(N+3 / 2) m}
\end{aligned}
$$

This finishes the proof of (5.13).

Remember that by construction of $P^{N, M}\left(\mathscr{P}_{i j}\right)=\phi\left(\mathscr{P}_{i j}\right), i=M, \ldots, M+N$, $j=M, \ldots, M+N$. Hence (5.13) implies

$$
\left|P^{N}\left(\mathscr{P}_{i j}\right)-\phi\left(\mathscr{P}_{i j}\right)\right| \leqq K\left|\mathscr{P}_{i j}\right|^{N+3 / 2} \text {. }
$$

Using (5.7), we can bound $\left|P^{N}(x)-\phi(x)\right| \leqq K|x|^{N+3 / 2} \quad$ whenever $x \in W_{\mathscr{P}_{i j}}^{\mathrm{s}, \delta} \cup W_{\mathscr{P}_{1}}^{\mathrm{u}, \delta}$ with $\delta=\frac{1}{2}\left|\mathscr{P}_{i j}\right|$. From that, using Corollary 5.3 we can conclude that $|\phi(y)-P(y)| \leqq K|y|^{N+3 / 2}$ when $|y|<\beta, y \in \Omega_{\varepsilon}$.

In effect, if $y \in \Omega_{\varepsilon}$, by Corollary 5.3 we know that

$$
W_{y}^{\mathrm{s}, \delta} \cap W_{\left(x_{i}^{1}, x_{j}^{2}\right)}^{\mathrm{u}, \delta} \quad W_{y}^{\mathrm{u}, \delta} \cap W_{\left(x_{i}^{1}, x_{j}^{2}\right)}^{\mathrm{s}, \delta}
$$

for all of the $x_{i}^{2} x_{j}^{2}$ in the grid converging to that we used to construct the interpolating polynomial.

We can consider $\mathscr{G}\left(N, M, N, M^{\prime}\right)$ in such a way that $y$ is the boundary element. That is, we consider $x_{M}^{1}, \ldots, x_{M+N+1}^{1}, x_{M^{\prime}}^{2}, \ldots, x_{M^{\prime}+N+1}^{2}$ in such a way that $W_{y}^{\mathrm{s}, \delta}$ intersects each of $W_{x_{i}^{1} x_{j}^{2}}^{\mathrm{u},}$ and $W_{y}^{\mathrm{u}, \delta}$ intersects each of $W_{x_{i}^{2}}^{\mathrm{s}, \delta}$. Moreover, using the uniformity in Corollary 5.3, we can find a constant such that given $y \in \Omega_{(\varepsilon)},|y|<\beta$ it is possible to find $\left\{\left(x_{i}^{1} x_{j}^{2}\right) i=M, M+1, \ldots, M+N, j=M^{\prime}, M^{\prime}+1, \ldots\right.$, $\left.M^{\prime}+N\right\}$ such that $d\left(y, \mathscr{P}_{i j}\right) \leqq K|y|$.

We now consider $|\phi-\bar{P}|_{W_{y}^{\text {s. }} \text {. }}$ By the estimates in (5.8) we have that this function, evaluated at the $N+1$ points $W_{y}^{\mathrm{s}, \delta} \cap W_{\mathscr{P}_{i j}}^{\mathrm{u}, \delta}$ is smaller than $K|y|^{N+2}$.

By the assumption that $\phi \in C_{\mathrm{s}}^{N+2}$ and the estimates on $P$ we have that $f-\left.\phi\right|_{W_{Y}^{\text {s. }}}$ is $C^{N+2}$ and we have bounds on the $C^{N+2}$ norm, e.g., if we parametrize it by the coordinates.

We claim that since this function is $C^{N+2}$, and is small at $N+1$ points it should be small in the middle.

Proposition 5.12. Let $x_{0}<\cdots<x_{N}$ be points in the line. Assume that

$$
\frac{x_{0}-x_{N}}{x_{i}-x_{i+1}} \leqq K \quad\left|\phi\left(x_{i}\right)\right| \leqq \varepsilon \quad i=0, \ldots, N
$$


Then

$$
|\phi(x)| \leqq\left[\varepsilon+\left|x_{0}-x_{N}\right|^{N+2}\|\phi\|_{C^{N+2}}\right] K^{\prime},
$$

where $K^{\prime}$ depends only on $K$.

Proof. We denote by $K^{\prime}$ all constants that depend only on $N K$.

By Proposition 5.8 we can construct a polynomial $P$ such that

$$
P\left(x_{i}\right)=\phi\left(x_{i}\right) \text {. }
$$

By (5.3) we have

$$
\|P\|_{C^{N+2}} \leqq K^{\prime} \varepsilon .
$$

Therefore $\phi-P$ vanishes at $N+1$ points and its $(N+2)$ derivative is bounded by $\|\phi\|+K^{\prime} \varepsilon$. Since obviously the Lagrange interpolation polynomial for $\phi-P$ is zero, if we had proved the estimates (5.2) for the whole space, then we could apply the easy version of the converse to Taylor's theorem that can be found in [AR, Gr].

Unfortunately, for general closed sets we need the full Whitney extension theorem whose hypothesis requires

$$
\left|\phi(y)-\phi(x)-\left(P_{x}^{N}(y)-P_{x}^{N}(z)\right)\right| \leqq K|y-z|^{N+2}
$$

as $y, z \rightarrow x$. See [R] for an example where the extension theorem fails even if the hypothesis we have established so far holds. So, we strengthen the arguments to verify the Whitney condition. quely,

We observe that, using Corollary 5.3 we can find $[y, z]=W_{y}^{\mathrm{s}, \delta} \cap W_{z}^{\mathrm{u}, \delta}$ uni-

$$
\begin{aligned}
\left|\phi(y)-\phi(z)-\left(P_{x}^{N}(y)-P_{x}^{N}(z)\right)\right| \leqq & \left|\phi(y)-P_{x}^{N}(y)-\phi([y, z])-P_{x}^{N}([y, z])\right| \\
& +\left|\phi(z)-P_{x}^{N}(z)-\phi([y, z])-P_{x}^{N}([y, z])\right| .
\end{aligned}
$$

The first term can be bound by $\left\|\phi-P^{N}\right\|_{C_{s}^{N+2}}|y-[y, z]|^{N+2}$ and the second

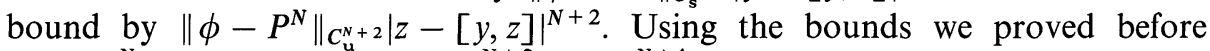
$\left\|\phi-P^{N}\right\|$ is bounded both in $C_{\mathrm{s}}^{N+2}$ and $C_{\mathrm{u}}^{N+1}$. Moreover, using Corollary 5.3 we have that

$$
|y-[y, z]|+|z-[y, z]| \leqq K|y-z| .
$$

Hence, we are done. This finishes the proof of Theorem 1.1.

This theorem applies without any modification to any other geometric objects, e.g. homeomorphisms that, by taking coordinates, can be reduced to sets of functions.

To conclude the proof of Theorem 1.3, the only thing that remains to be proved is the following proposition from elementary measure theory which verifies that a large subset of $\Omega_{\varepsilon}$ consists of points that verify condition iv) of Theorem 5.7:

Proposition 5.13. Let $\varepsilon$ be any real number bigger than zero. Let $\Omega_{\varepsilon}$ be a set as in Theorem 5.1. Then, for any $\varepsilon^{\prime}>\varepsilon$ we can find a set $\Lambda_{\varepsilon^{\prime}}$ in such a way that

1) $\Lambda_{\varepsilon^{\prime}} \subset \Omega_{\varepsilon}$.

2) Its complement has measure less than $\varepsilon^{\prime}$.

3) $\Lambda_{\varepsilon^{\prime}}$ is closed.

4) For any $\gamma, \gamma^{\prime}$ and all $\beta$ small enough, the condition iv) of Theorem 5.7 holds for all points in $\Lambda_{\varepsilon^{\prime}}$. 
Proof. Let $x$ be a density point of $\Omega_{\varepsilon}$. Make a differentiable change of coordinates in such a way that $x$ is situated at the origin and the stable and unstable manifolds are the coordinate axis. If $y$ is a point, we will denote its coordinates by $y^{1}, y^{2}$.

We claim that for some conveniently chosen $1<\gamma<\gamma^{\prime}$, we can find a sequence $\left\{x_{n}\right\}$ converging to $x$ in such a way that all $\gamma<x_{n}^{1} / x_{n+1}^{1}<\gamma^{\prime} \gamma<x_{n}^{2} / x_{n+1}^{2}<\gamma^{\prime}$.

In effect, fix numbers $\gamma_{1}<1<\gamma_{2}$. By the theorem of differentiation of integrals by rectangles (see e.g. [Gu]) there is a subset - the subset of "points of density" - of $\Omega_{\varepsilon}$ with the same measure as $\Omega_{\varepsilon}$ all of whose points have the following property: If $O$ is a point of density of the set $\Omega_{\varepsilon}$, for any given $\varepsilon_{1}>0$, we can find numbers $a^{*}, b^{*}$ such that for any rectangle $\mathscr{R}_{a b}$ with corner at $O$, width $a$ and height $b$ such that $a<a^{*}, b<b^{*}, \gamma_{2} b>a<\gamma_{1} b$, then $\mu\left(\mathscr{R}_{a b} \cap \Omega_{\varepsilon}\right) \geqq\left(1-\varepsilon_{1}\right) \mu\left(R_{a b}\right)$, where $\mu$ is a measure equivalent to Lebesgue.

We also make the elementary observation that, for all $\mathscr{R}_{a b}$ with $\gamma_{2} b>a<\gamma_{1} b$ denoting by $\widetilde{\mathscr{R}}_{a b}$ the set $\left\{x \in \mathscr{R}_{a b} \mid \gamma_{2} x^{2}>x^{1}<\gamma_{1} x^{2}, a / \gamma^{\prime}<x^{1}<a / \gamma, b / \gamma^{\prime}<x^{2}<b / \gamma\right\}$, we have that $\mu \tilde{R}_{a b} \geqq \kappa \mu \mathscr{R}_{a b}$ with $\kappa$ a constant that depends only on $\gamma, \gamma^{\prime}, \gamma_{1}, \gamma_{2}$ and which for some appropriate choices is strictly positive. Given any $\gamma, \gamma^{\prime}, \kappa$ can be made strictly positive by taking $\gamma_{1}, \gamma_{2}$ close enough to 1 .

Now we can construct the sequence converging to $O$ claimed in Proposition 5.13 as follows: Pick the $\gamma^{\prime}$ 's in such a way that $\kappa$ is strictly positive and find $a^{*}, b^{*}$ corresponding to $\varepsilon_{1}=\kappa / 2$. Choose a point $x_{0} \in \Omega$ such that $0<x_{0}^{1}<a^{*}$, $0<x_{0}^{2}<b^{*}, \gamma_{2} x_{0}^{2}>x_{0}^{1}<\gamma_{1} x_{0}^{2}$ (such points can be shown to exist using the theorem of differentiation of integrals). We assume inductively that we have obtained points $\left\{x_{i}\right\}_{i=0}^{N}$ satisfying $i<x_{i}^{1}<a^{*}, i<x_{i}^{2}<b^{*}, \gamma_{2} x_{i}^{2}>x_{i}^{1}<\gamma_{1} x_{i}^{2}$.

Then the set $\tilde{\mathscr{R}}_{x_{N}^{1} x_{N}^{2}} \cap \Omega_{\varepsilon}$ has positive measure, in particular it is not empty. Choose $x_{N+1}$ any point in the set. By construction, the coordinates of $x_{N+1}$ satisfy the inductive bounds. It is also easy to check that the ratios needed in the claim are also propagated. This establishes the claim.

Using the fact that the stable manifold depends continuously on the point, we can check that if we fix $\gamma, \gamma^{\prime}$ a point of density will satisfy the conditions iv) of Theorem 5.7 for some $\beta$. Therefore, taking $\beta$ sufficiently small, we can ensure that the set that safisfies the conditions has measure as close to that of $\Omega_{\varepsilon}$ as desired.

We also observe that a property of Lebesgue measure is that the measure of a set is the supremum of the measures of its closed subsets. Therefore, we can assume that the set for which Proposition 5.13 applies is closed.

The argument given so far proves Theorem 1.3 for any $k \in \mathbf{N}$. To prove Theorem 1.3 with $k=\infty$ we observe that for any $r \in \mathbf{Z}$, we can obtain closed sets whose complements has measure less than $\varepsilon 2^{-k}$ on which $h$ is $C^{r}$. On the intersection of these sets, $h$ is $C^{\infty}$.

This finishes the proof of Theorem 1.3 under hypothesis i). To prove it under hypothesis ii), we just observe that it suffices to show that the only property of $h$ we have used is that it is continuous and that it transforms a measure satisfying Pesin's entropy formula into another one that also satisfies it.

Unfortunately, it seems quite difficult to prove an analogous result using invariants at periodic orbits. Notice that, for example, it is not clear even that a system with positive Lyapounov exponents has only countably many periodic orbits.

There are arguments in the literature that could imply that measures with nonzero Lyapounov exponents can be approximated in the weak sense by measures concentrated on periodic orbits. Nevertheless, it is not clear that the 
Lyapounov exponents on these measures approximate those of the limiting measure. As it is well known, they should be lower semicontinuous with respect to taking weak limits on the measure, but it is discontinuous. (I am grateful to F. Ledrapier and L.-S. Young for explaining these examples to me.)

\section{Extensions to High Dimensions}

In this section, we show that there are several extensions of this circle of ideas to higher dimensions, but that the situation is intrinsically more complicated as evidenced by counterexamples.

6.1. Results in Higher Dimensions. The results of the previous sections can be described as saying that if some Anosov diffeomorphisms are equivalent in some sense, they are actually equivalent in an stronger sense. These results are usually described under the name rigidity.

We will prove that all $C^{\infty}$ Anosov diffeomorphisms and flows have the property that sufficiently smooth conjugacies between them are $C^{\infty}$. More precisely

Theorem 6.1. Let $f, g$ be two $C^{r}, r \in \mathbf{N} \cup\{\infty\}$ Anosov diffeomorphisms (respectively $\sigma_{t}, \psi_{t}$ be two Anosov flows) on a compact manifold. Then, there exists a $\kappa(f) \in \mathbf{N}$ (respectively $\kappa\left(\sigma_{t}\right)$ ) such that if

$$
h \circ f=g \circ h \quad\left(\text { respectively } h \circ \sigma_{r}=\psi_{t}\right) \text {, }
$$

and $h, h^{-1}$ are $C^{\kappa}$, then $h, h^{-1}$ are $C^{r-\varepsilon}$. Moreover, $\kappa(f)$ can be chosen to be constant in $C^{1}$ neighborhoods of Anosov diffeomorphisms.

Remark. Unfortunately, we do not know how to prove an analogous result for $k=\omega$ except in the case that the manifold is two dimensional (See [L12].)

Remark. Notice that since $h \circ f^{n}=g^{n} \circ h$, we have $\kappa(f) \leqq \kappa\left(f^{n}\right)$.

Proof. We will present the proof for diffeomorphisms only since the proof for flows only requires a change of notation.

We recall that by the invariance of the stable and unstable bundles, we can write $D f(x)=D f_{\mathrm{s}}(x) \oplus D f_{\mathrm{u}}(x)$, where $D f_{\mathrm{s}}(x): E_{x}^{\mathrm{s}(f)} \mapsto E_{f(x)}^{\mathrm{s}(f)}, \quad D f_{\mathrm{u}}(x): E_{x}^{\mathrm{u}(f)} \mapsto E_{f(x)}^{\mathrm{u}(f)}$, and analogously for $g$.

Moreover, if $h$, satisfying (6.1) is $C^{1}$, we have $D f \circ h D h=D h \circ g D g$ and, hence, we also have a decomposition $D h=D h_{\mathrm{s}} \oplus D h_{\mathrm{u}}$, where $D h_{\mathrm{s}}(g(x)): E_{g(x)}^{\mathrm{s}(g)} \mapsto E_{h(x)}^{\mathrm{s}(f)}$, $D h_{\mathrm{u}}(g(x)): E_{g(x)}^{\mathrm{u}(g)} \mapsto E_{h(x)}^{\mathrm{u}(f)}$.

We claim that we can take $\kappa$ to be any number bigger than 1 for which it is possible to find $C>0, \lambda<1$ such that:

$$
\begin{array}{ll}
\sup _{x \in M}\left\|D f_{\mathrm{s}}^{n}(x)\right\|^{\kappa}\left\|D f_{\mathrm{s}}^{-n}(x)\right\| \leqq C \lambda^{n} & n>0, \\
\sup _{x \in M}\left\|D f_{\mathrm{u}}^{n}(x)\right\|^{\kappa}\left\|D f_{\mathrm{u}}^{-n}(x)\right\| \leqq C \lambda^{-n} & n<0 .
\end{array}
$$

Remark. Notice that the value of $\kappa$ defined by (6.2) depends only on the Lyapounov exponents of $f$. 
Remark. 'Notice that, using Mather's characterization of Anosov diffeomorphisms, [Mat] the above relations are satisfied if the spectrum of the push forward $f_{*}$ acting on continuous vector fields is contained in $\left\{z \in \mathbf{C}\left|r^{\kappa}-\varepsilon<\right| z \mid<r\right\} \cup\left\{z \in \mathbf{C} \mid r^{\prime}<\right.$ $\left.|z|<r^{\prime \kappa}\right\}$ for some $0<r<1<r^{\prime}$.

Remark. Notice also that the spectrum of $f_{*}$ is an invariant of $C^{1}$ conjugacy so that, provided that we take $\kappa>1$, we can assume that the hypotheses are verified both by $f$ and $g$.

It can be verified by a direct calculation that, if $f$ and $g$ are $C^{1}$ conjugate, then $\sup _{x \in M}\left\|D f^{n}(x)\right\|<C \sup _{x \in M}\left\|D g^{n}(x)\right\|$. Actually, the way that the conditions enter in the proof are really between the spectrum of $f$ and the spectrum of $g$.

The fact that $\kappa$ can be chosen constant in open neighborhoods follows from the well known fact that the spectrum of $f_{*}$ acting on continuous functions depends continuously on $f$ in the $C^{1}$ topology. It follows for a careful reading of most of the proofs of the $C^{1}$ openness of the set of Anosov diffeomorphisms. See for example [Sh], Prop. 7.6. (It is a non-trivial statement since the map that to $f$ assigns $f_{*}$ is discontinuous if we topologize $f_{*}$ with the norm on operators on continuous functions and $f$ with the $C^{1}$ topology.)

To prove Theorem 6.1 we will show that if $h \in C_{\mathrm{s}}^{k}(g)$ and $k \geqq \kappa$, then $h \in C_{\mathrm{s}}^{k+1}(g)$. Notice that, since $h$ maps leaves of the stable foliation of $g$ into leaves of the stable foliation of $f$, we can restrict (6.1) to stable leaves of $f$. Denoting by $D_{\mathrm{s}(f)}$ the derivative of the restriction of a function to the leaves of the stable foliation of $f$, we obtain differentiating the restriction of (6.1) to the stable manifolds of $g$,

$$
D_{\mathrm{s}(g)}^{k} h(x)=\left[D_{\mathrm{s}} f \circ h\right]^{-1}\left(D_{\mathrm{s}(g)}^{k} h\right) \circ g(x)\left[D_{\mathrm{s}} g\right]^{\otimes k}+R(x),
$$

where $R(x)$ is a polynomial in the stable derivatives of $h$ of order up to $k-1$. In particular, it is a function in $C_{\mathrm{s}(g)}^{1}$. That is, there is an explicit formula for $D_{\mathrm{s}(g)}^{k} h(x)$ in terms of $D_{\mathrm{s}(g)}^{k} h(g(x))$ and lower order derivatives of $h$.

We observe that, applying repeatedly (6.3) and the chain rule, we obtain:

$$
\begin{aligned}
D_{\mathrm{s}(g)}^{k} h(x)= & {\left[\left(D_{\mathrm{s}} f\right) \circ h\right]^{-1} \circ g^{N+1}(x) h \circ g^{N+1}(x)\left[D_{\mathrm{s}} g\right]^{\otimes k} \circ g^{N+1}(x) } \\
& +\sum_{j=0}^{N}\left(\prod_{i=0}^{j-1}\left[\left(D_{\mathrm{s}} f\right) \circ h\right]^{-1} \circ g^{j-1-i}\right)(x) R \circ g^{j}(x)\left(\prod_{i=0}^{j-1}\left[D_{\mathrm{s}} g\right]^{\otimes k} \circ g^{i}\right)(x) \\
= & {\left[D_{\mathrm{s}} f \circ f^{N+1} \circ h\right]^{-1} \circ g^{N+1}(x) h \circ g^{N+1}(x)\left[D_{\mathrm{s}} g\right]^{\otimes k} \circ g^{N+1}(x) } \\
& +\sum_{j=0}^{N}\left[\left(D_{\mathrm{s}} f^{j}\right) \circ h\right]^{-1}(x) R \circ g^{j}(x)\left[D_{\mathrm{s}} g^{j}\right]^{\otimes k}(x) .
\end{aligned}
$$

Using (6.2), we see that the first term tends to zero uniformly in $x$, so that:

$$
D_{\mathrm{s}(g)}^{i} h(x)=\sum_{j=0}^{\infty}\left[\left(D_{\mathrm{s}} f^{j}\right) \circ h\right]^{-1}(x) R \circ g^{j}(x)\left[D_{\mathrm{s}} g^{j}\right]^{\otimes k}(x)
$$

Taking derivatives term by term in the series in (6.4), we obtain:

$$
\begin{aligned}
& \sum_{j=0}^{\infty} D_{\mathrm{s}}\left(\left[\left(D_{\mathrm{s}} f^{j}\right) \circ h\right]^{-1}\right)(x) R \circ g^{j}(x)\left[D_{\mathrm{s}} g^{j}\right]^{\otimes k} \\
& \quad+\left[\left(D_{\mathrm{s}} f^{j}\right) \circ h\right]^{-1}(x)\left(D_{\mathrm{s}(g)} R\right) \circ g^{j}(x)\left(D_{\mathrm{s}(g)} g^{j}\right)(x)\left[D_{\mathrm{s})} g^{j}\right]^{\otimes k} \\
& \quad\left[\left(D_{\mathrm{s}} f^{j}\right) \circ h\right]^{-1}(x) R \circ g^{j}(x) D_{\mathrm{s}}\left[D_{\mathrm{s}} g^{j}\right]^{\otimes k} .
\end{aligned}
$$


Using that:

$$
\begin{aligned}
D_{\mathrm{s}}\left[D_{\mathrm{s}} g^{j}\right]^{\otimes k} & =\sum_{i=1}^{k} D_{\mathrm{s}} g^{j} \otimes \cdots i \cdots \otimes D_{\mathrm{s}} g^{j} D_{\mathrm{s}}^{2} g^{j} \otimes \cdots \otimes D_{\mathrm{s}} g^{j} \\
D_{\mathrm{s}}^{2} g^{j} & =D_{\mathrm{s}} \prod_{i=1}^{j} D_{\mathrm{s}} g \circ g^{i} \\
& =\sum_{i=1}^{j} D_{\mathrm{s}} g \circ g(x) \cdots D_{\mathrm{s}} g \circ g^{i-1} D_{\mathrm{s}}^{2} g \circ g^{i}\left(D_{\mathrm{s}} g^{i}\right) D_{\mathrm{s}} g^{i+1} \cdots D_{\mathrm{s}} g^{j}
\end{aligned}
$$

$$
\begin{aligned}
D_{\mathrm{s}}\left(\left[\left(D_{\mathrm{s}} f^{j}\right) \circ h\right]^{-1}\right)(x)= & D_{\mathrm{s}} \prod_{i=1}^{j}\left[D_{\mathrm{s}} f\right]^{-1} \circ f^{-} i \circ h(x) D_{\mathrm{s}(g)} h(x) \\
= & \sum_{i=0}^{j}\left(D_{\mathrm{s}} f^{-1}\right) \circ f^{-1} \circ h(x) D_{\mathrm{s}(g)} h(x) \\
& +\prod_{i=1}^{j}\left[D_{\mathrm{s}} f\right]^{-1} \circ f^{-} \circ h(x)\left[D_{\mathrm{s}(g)}\right]^{2}
\end{aligned}
$$

as well as the bounds (6.2), we obtain that the sum in (6.5) satisfies the conditions of Weirstrass' $M$ test so that it converges uniformly on the $M$. Therefore, it is continuous in $M$ and it is a bona-fide derivative of (6.4). Hence $h$ is in $C_{\mathrm{s}(g)}^{k+1}$ as claimed. The process can be iterated till we conclude that $h \in C_{\mathrm{s}(g)}^{r}$.

A similar argument with $f$ and $g$ replaced by $f^{-1}, g^{-1}$ will prove that $h \in C_{\mathrm{s}(g)}^{r}$. Using Theorem 5.7, the Theorem 6.1 is established.

The same argument works for non-uniformly hyperbolic systems.

Theorem 6.2. Let $f$ be $C^{r}$ diffeomorphism, $r \in \mathbf{N} \cup\{\infty\}$ on a compact manifold $M$, preserving a measure $\mu^{(f)}$ equivalent to Lebesgue and such that it is ergodic with respect to $\mu^{(f)}$ and it has no zero Lyapounov exponents. It is possible to find a $\kappa$ depending only on the Lyapounov exponents of $f$ such that if $f, g, h$ satisfy (6.1) and $h$ is $C^{\kappa}$. Then, for every $\varepsilon>0$, we can find a closed set $\Omega_{\varepsilon} \subset M$ whose complement has Lebesgue measure less than $\varepsilon$ such that $\left.h\right|_{\Omega_{\varepsilon}} \in C^{r-\varepsilon}$.

Proof. We just observe that, if $\Omega_{\varepsilon}$ is a "good" set as in Theorem 5.1 the arguments in the proof of Theorem 6.1 to bootstrap the regularity in the stable and unstable manifolds work exactly the same on the local stable and unstable manifolds of this set.

We can verify as in Proposition 5.13 that a subset of $\Omega_{\varepsilon}$ of measure as close to that of $\Omega_{\varepsilon}$ as we like will have the exponential approximation property by points of $\Omega_{\varepsilon}$ and, hence the proof of Theorem 5.7 goes through without change.

6.2. Counterexamples. In this subsection, for every $k \in \mathbf{N}$ we construct pairs of analytic Anosov diffeomorphisms in $T^{d}, d \geqq 4$ which are $C^{k}$ conjugated but they are not conjugated by any $C^{k+1}$ diffeomorphism. They are thus, counterexamples to several extensions of the theorems we considered in the previous sections. They are also counterexamples to similar extensions of related results.

For example, the systems we construct here have analytic stable and unstable manifolds yet they are not analytically conjugate to linear automorphisms of the 
torus. Flaminio and Katok showed in [FK] that all Anosov diffeomorphisms in $T^{2}, T^{3}$ whose invariant stable and unstable foliations are $C^{\infty}$ conjugate to linear automorphisms. The examples here are also counterexamples to extensions of the results of $[\mathrm{LM}]$ on Anosov diffeomorphisms on $T^{2}$ to higher dimensions.

In [LM] it was shown that if two $C^{\infty}$ Anosov diffeomorphisms of $T^{2}$ are topologically conjugate and the Lyapounov exponents at corresponding periodic orbits agree, the conjugating diffeomorphism is $C^{\infty}$. The diffeomorphisms we construct in this section not only have the same Lyapounov exponents at periodic orbits but also several other invariants of differentiable conjugacy vanish. The stable eigenvalues of the return map cannot be expressed as the product of other stable eigenvalues. Hence, by the Sternberg linearization theorem, the restrictions of the return map to the stable (respectively unstable) manifolds of corresponding periodic orbits are $C^{\infty}$ conjugate in small enough neighborhoods. This shows that in dimensions higher than 4 there is no set of local invariants associated to periodic orbits that guarantee that all conjugacies between the diffeomorphisms are $C^{\infty}$.

As far as we know, the situation is not settled for diffeomorphisms of $T^{3}$.

Theorem 6.3. For any $k \in \mathbf{N}$ and any $d \geqq 4$, there exist $f, g$ Anosov diffeomorphisms of $T^{d}$ and an $h, a C^{k}$ diffeomorphism of $T^{d}$ such that

$$
f \circ h=h \circ g
$$

and $h \notin C^{k+1}$.

Proof. We will consider $T^{d}=T^{2} \times T^{d-2}$ and write a point of $T^{d}$ as $(x, y)$ with $x \in T^{2}, y \in T^{d-2}$.

We will take $f$ of the form

$$
f(x, y)=(A x, B y)
$$

with $A, B$ Anosov linear automorphisms of $T^{2}$ and $T^{d-2}$ respectively and $g$ of the form

$$
g(x, y)=\left(A x, B(y)+e_{\mathrm{u}} \varphi(x)\right),
$$

where $e_{\mathrm{u}}$ is an unstable eigenvector of $B, \varphi: T^{2} \rightarrow \mathbf{R}$ is a trigonometric polynomial, which we assume is just $\cos \left(x_{1}\right)$.

If $\varphi$ is small enough, we can invoke the structural stability theorem to obtain an $h$ which is close to the identity. This $h$ will be unique among all the homeomorphisms $C^{0}$ close to the identity.

It is possible to obtain a representation of $h$ by trying an $h$ of the form.

$$
h(x, y)=\left(x, y+e_{\mathrm{u}} \psi(x)\right) .
$$

If we succeed in finding a solution of (6.8), we can use the uniqueness part of structural stability to conclude that it is the conjugacy. We will show that, by imposing conditions on the spectrum of $A$ and $B$, we can get an $h$ with the desired regularity properties.

Substituting (6.8) into (6.7), we obtain the following equation for $\psi$ :

$$
B e_{\mathrm{u}} \psi(x)=\psi(A x) e_{\mathrm{u}}+e_{\mathrm{u}} \varphi(x) .
$$

Hence if $B e_{\mathrm{u}}=\lambda e_{\mathrm{u}}$ the equation becomes

$$
\lambda \psi(x)-\psi(A x)=\varphi(x)
$$


This equation can be conveniently analyzed using Fourier series.

If

$$
\begin{aligned}
& \varphi(x)=\sum \hat{\varphi}_{k} e^{2 \pi i k x}, \\
& \psi(x)=\sum \hat{\psi}_{k} e^{2 \pi i k x}
\end{aligned}
$$

in the sense of distributions, the conjugacy equation becomes

$$
\lambda \hat{\psi}_{k}-\hat{\psi}_{C k}=\hat{\varphi}_{k}
$$

where $C=\left(A^{-1}\right)^{t}$.

If we choose $\hat{\psi}_{k_{0}}$ for some $k_{0}$, the equation determines $\hat{\psi}_{k}$ for all $k$ in the orbit of $k_{0}$ under $C$. The choice of $\hat{\psi}_{k_{0}}$ will be determined by imposing that $\left|\hat{\psi}_{k}\right| \rightarrow 0$ as $|k| \rightarrow \infty$. We now argue that such choices are sometimes possible.

If $\varphi$ has any one Fourier components $\hat{\varphi}_{k_{0}}$ different from zero (and of course $\left.\hat{\varphi}_{-k_{0}}=\hat{\varphi}_{k_{0}}^{*}\right)$ then we can find a $\psi$ by setting $\hat{\psi}_{k}=0$ for all $k \notin\left\{ \pm C^{n} k_{0}\right\}_{n=-\infty}^{\infty}$.

For $k= \pm C^{n} k_{0}, n \geqq 1$ we set $\hat{\psi}_{k}=0$. For $n \leqq 0$ we set $\hat{\psi}_{ \pm C^{n} k_{0}}= \pm \lambda^{-n} \hat{\Phi} \pm k_{0}$.

These choices lead to a solution of the equation. Since $\sum\left|\hat{\psi}_{k}\right|<\infty$, the Fourier series converges uniformly and $\psi$ is a continuous function. Moreover we observe that $\psi$ is $C^{0}$ close to zero if $\left|\hat{\varphi}_{k_{0}}\right|$ is small.

If we denote the eigenvalues of $A$ by $\mu, 1 / \mu,|\mu|>1$ we claim that

$$
\psi \in \Lambda_{\alpha} \quad \text { when } \quad \alpha<\frac{\log \mu}{\log \lambda} \equiv \alpha_{C}
$$

and does not belong to any $\Lambda_{\alpha}$ with $\alpha>\alpha_{C}$, where $\Lambda_{\alpha}$ denotes the Lipschitz space (see St, Kr]) which agrees with the usual Hölder spaces for $\alpha \in \mathbf{N}$ and, for $\alpha \in \mathbf{N}$, $\Lambda_{\alpha} \supset C^{k}$. The claim follows from the characterization of $\Lambda_{\alpha}$ spaces by the size of their Fourier coefficients that can be found in [St] or [Kr]. Notice that $\left|C^{n} k_{0}\right| \geqq K \mu^{n}$. Hence, the non-zero Fourier coefficients of $\psi$ are exponentially far apart.

If we take $M, N$ hyperbolic automorphisms of $T^{2}$ and $T^{d-2}$ we observe that taking $A=M^{n}, B=N^{m}, \alpha_{C}=\frac{n}{m} \frac{\log \left|\mu_{0}\right|}{\log \left|\lambda_{0}\right|}$ where $\lambda_{0}, \mu_{0}$ denote the eigenvalues of $M, N$ of largest and smallest absolute value, so that, by choosing $n, m$, we can arrange to have $\alpha_{C}$ in any point of a set dense in $\mathbf{R}$. The claim of the theorem is thus established.

Remark. The fact that $\psi$ is in $\Lambda_{\alpha}, \alpha<\alpha_{C}$ can also be established by observing that iterating the cohomology equation we can write

$$
\psi(x)=\sum_{n=0}^{\infty} \lambda^{-(n+1)} \varphi\left(A^{n} \kappa\right)
$$

It is possible to use the chain rule to estimate the $\Lambda_{\alpha}$ norm of the terms of the series and show that the series converges uniformly in $\Lambda_{-\alpha}$ when $\alpha<\alpha_{C}$.

Remark. Since $w_{h(x)}^{\mathrm{u}(y)}=h\left(w_{\kappa}^{\mathrm{u}(f)}\right), w_{h(x)}^{\mathrm{s}(g)}=h\left(w_{\kappa}^{\mathrm{s}(f)}\right)$ and the stable/unstable foliations of $f$ are analytic, we see that the stable/unstable foliations of $g$ are at least as differentiable as $\psi$.

Actually, using the explicit formula for $h$, we can see that $h$ maps an unstable leaf for $f$ into another unstable leaf for $f$. Hence, the unstable foliation for $g$ is also 
analytic. This is in contrast with the result of $[\mathrm{FK}]$ who showed that the fact that one foliation of an analytic diffeomorphism of $T^{2}$ is analytic implies that it is analytically conjugate to a linear automorphism. On the other hand, the stable foliation is not more differentiable than $\psi$. This, again, can be seen by using the explicit form for $h$ and computing derivatives along unstable directions in $T^{2}$ of the stable leaves.

Remark. The same idea can be used to construct similar examples in manifolds other than tori. One can use as product diffeomorphisms linear automorphisms of nilmanifolds. In that case, the argument of lack of regularity is slightly more complicated since one cannot compute with Fourier coefficients.

\section{References}

[AR] Abraham, R., Robbin, J.: Transversal mappings and flows. N.Y.: Benjamin 1967

[A] Anosov D.V.: Geodesic flows on closed Riemannian manifolds with negative curvature. Proc. Stek. Inst. 90, 1-235 (1967)

[BR] Bowen, R. Ruelle, D.: The ergodic theory of Axiom A flows. Invent. Math. 29, 181-202 (1975)

[Bo] Bowen, R.: Periodic points and measures for Axiom A diffeomorphisms. Trans. A.M.S. 154, 377-398 (1971)

[Bo2] Bowen, R.: The equidistribution of closed geodesics. Am. J. Math. 94, 413-423 (1972)

[Ca] Campanato, S.: Propietá di una famiglia di spazi funzionali. Ann. Scuo. Norm. Pyer 18, 137-160 (1964)

[FHY] Fathi, A., Herman, M., Yoccoz, J-C.: A proof of Pesin's stable manifold theorem. Geometric Dynamics, J. Palis (ed.), Lect. Notes in Math. vol. 1007, Berlin, Heidelberg, New York: Springer 1983

[FK] Flaminio, L., Katok, A.: Rigidity of symplectic Anosov diffeomorphisms on low dimensional tori. Ergod. Th. \& Dyn. Sys. 11, 427-41 (1991)

[G1] Glaeser, G.: Etude de quelques algèbres Tayloriennes. J. Anal. Math. 11, 1-118 (1958)

[Gu] de Guzman, M.: Differentiation of integrals in $\mathbf{R}^{n}$. Lect. Notes in Math. vol. 481, Berlin, Heidelberg, New York: Springer

[HK] Hurder, S., Katok, A.: Differentiability, rigidity and Godbillon-Vey classes for Anosov flows. Pub. Mat. I.H.E.S. 72, 5-61 (1990)

[HPS] Hirsch, M., Pugh, C., Shub, M.: Invariant manifolds. Lect. Notes in Math. vol. 583, Berlin, Heidelberg, New York: Springer 1977

[HP] Hirsch, M., Pugh, C.: Stable manifolds and hyperbolic sets. Proc. Symposia in Pure Math. 14, 133-163 (1969)

[He] Herman, M.: Sur la conjugaison différentiable des difféomorphismes du cercle a des rotations. Pub. Mat. I.H.E.S. 49, 5-234 (1979)

[Jo1] Journé J-L.: On a regularity problem occurring in connection with Anosov diffeomorphisms. Commun. Math. Phys. 106, 345-352 (1988)

[Jo2] Journé, J-L.: A regularity lemma for functions of several variables. Rev. Mat. Iber. 4, 187-193. (1988)

[FO] Feldman, J., Ornstein, D.: Semi-rigidity of horocycle flows over compact surfaces of variable negative curvature. Erg. Theo. Dyn. Sys. 7, 49-72 (1987)

[Ka] Katok, A.: Lyapounov exponents, entropy and periodic orbits for diffeomorphisms. Pub. Mathematiques du I.H.E.S. 51, 137-174 (1980)

[Kr] Krantz, S.: Lipschitz spaces, smoothness of functions and approximation theory. Expo. Mat. 3, 193-260 (1983)

[LMM] de la Llave, R., Marco, J.M., Moriyón, R.: Canonical perturbation theory for Anosov systems and regularity properties of Livsic's cohomology equation. Ann. Math. 123, 537-611 (1986) 
[LM] de la Llave, R., Moriyón, R.: Invariants for smooth conjugacy of hyperbolic dynamical systems IV. Commun. Math. Phys. 116, 185-192 (1988)

[LS] Ledrapier, F., Strelcyn, J-M.: A proof of the estimation from below in Pesin's entopy formula. Ergodic Theoret. Dyn. Syst. 2, 203-220 (1982)

[LY1] Ledrapier, F., Young, L-S.: The metric entropy of diffeomorphisms I: Characterization of measures satisfying Pesin's formula. Ann. Math. 122, 509-539 (1985)

[LY2] Ledrapier, F., Young, L-S.: The metric entropy of diffeomorphisms II: Relations between entropy, exponents and dimension. Ann. Math. 122, 540-574 (1985)

[Le] Ledrapier, F.: Propriétés ergodiques des mesures de Sinai. Pub. Mat. I.H.E.S. 59, 163-188 (1984)

[L11] de la Llave, R.: Invariants for smooth conjugacy of hyperbolic dynamical systems II. Commun. Math. Phys. 109, 369-378 (1987)

[L12] de la Llave, R.: Analytic regularity of solutions of Livsic's cohomology equation and applications to smooth conjugacy of hyperbolic dynamical systems. To appear in Ergodic Theoret. Dyn. Syst. (1987)

[L13] de la Llave, R.: New invariant manifold theorems and applications to smooth conjugacy of hyperbolic systems. preprint

[Ma] Margulis, G.: Certain measures associated with U-flows on compact manifolds. Funct. Anal. Appl. 4, 55-67 (1970)

[Mat] Mather, J.: Characterization of Anosov diffeomorphisms. Indag. Mat. 30, 479 (1969)

[Ne] Newhouse, S.: Lectures on Dynamical Systems. In: Dynamical Systems. C.I.M.E. Lectures 1978. Boston, Basel: Birkhauser 1980

[Pe] Pesin: Characteristic Lyapunov exponents and smooth ergodic theory. Russ. Math. Surv. 32, 55-114 (1977)

[Pl] Plante, J.: Anosov flows. Am. J. Math. 94, 729-754 (1972)

[Po1] Pollicott, M.: $C^{r}$-rigidity theorems for hyperbolic flows. Israel J. Math. 61, 14-27 (1988)

[Po2] Pollicott, M.: $C^{k}$-rigidity for hyperbolic flows. Israel J. Math. 69, 351-360 (1990)

[Pr] Pryzticki, F.: On holomorphic perturbations of $z \mapsto z^{n}$. Boll. Pol. Acad. Sci. 34, 127-132 (1986)

[PS] Pugh, C., Shub, M.: Ergodicity of Anosov actions. Invent. Math. 29, 7-38 (1975)

[PS2] Pugh, C., Shub, M.: Ergodic Atractors. I.B.M. preprint (1988)

[Ra] Rand, D.: Global phase-space universality, smooth conjugacies and renormalisation. The $C^{1+\alpha}$ case. Nonlinearity $1,181-202$ (1988)

[Ro] Robbin, J.: The Whitney extension theorem. in [AR]

[Ru] Ruelle, D.: A measure associated with Axiom A attractors. Am. J. Math. 98, 619-654 (1976)

[Ru2] Ruelle, D.: Ergodic theory of differentiable dynamical systems. Pub. Mathematiques du I.H.E.S. 50, 27-58 (1979)

[SS] Shub, M., Sullivan, D.: Expanding endomorphisms of the circle revisited. Ergodic Theoret. Dyn. Syst. 5, 285-290 (1985)

[Sh] Shub, M.: Stabilité globale des systèmes dynamiques. Asterisque 56 (1978)

[Si1] Sinai, Ja.G.: Gibbs measures in ergodic theory. Russ. Math. Surv. 27, 21-64 (1972)

[Si2] Sinai, Ja.G.: Dynamical systems with countably multiple Lebesgue spectrum II. A.M.S. Transl., Ser. 2, 68, 34-88 (1968)

[SB] Stoer, J., Burlish, R.: Introduction to Numerical Analysis. Berlin, Heidelberg, New York: Springer 1980

Communicated by J.-P. Eckmann 